\title{
A Unified Optimizing Compiler Framework for Different GPGPU Architectures
}

\author{
YI YANG and PING XIANG, North Carolina State University \\ JINGFEI KONG and MIKE MANTOR, Advanced Micro Devices \\ HUIYANG ZHOU, North Carolina State University
}

\begin{abstract}
This article presents a novel optimizing compiler for general purpose computation on graphics processing units (GPGPU). It addresses two major challenges of developing high performance GPGPU programs: effective utilization of GPU memory hierarchy and judicious management of parallelism. The input to our compiler is a naïve GPU kernel function, which is functionally correct but without any consideration for performance optimization. The compiler generates two kernels, one optimized for global memories and the other for texture memories. The proposed compilation process is effective for both AMD/ATI and NVIDIA GPUs. The experiments show that our optimized code achieves very high performance, either superior or very close to highly fine-tuned libraries.
\end{abstract}

Categories and Subject Descriptors: D.3.4 [Programming Languages]: Processors-Compilers, Optimization

General Terms: Performance, Experimentation, Languages

Additional Key Words and Phrases: GPGPU, OpenCL, CUDA, CUBLAS, GPU Computing

ACM Reference Format:

Yang, Y., Xiang, P., Kong, J., Mantor, M., and Zhou, H. 2012. A unified optimizing compiler framework for different GPGPU architectures. ACM Trans. Architec. Code Optim. 9, 2, Article 9 (June 2012), 33 pages.

DOI $=10.1145 / 2207222.2207225$ http://doi.acm.org/10.1145/2207222.2207225

\section{INTRODUCTION}

The high computational power and affordability of state-of-art graphics processing units (GPU) have made them the first widely accessible parallel computers with teraflops capability. To fully realize the power of general purpose computation on graphics processing units (GPGPU) or GPU computing, two key issues need to be considered carefully: (1) how to parallelize an application into concurrent work items and distribute the workloads in a hierarchy of thread blocks and threads and (2) how to efficiently utilize the GPU memory hierarchy, given its dominant impact on performance. As these two issues are usually coupled together and finding an optimal tradeoff between different levels of parallelism and memory optimizations requires detailed understanding of GPU hardware, developing high performance GPGPU

An earlier version of this article appeared in the ACM SIGNPLAN Conference on Programming Language Design and Implementation (PLDI'10) [Yang et al. 2010].

This work is supported by the National Science Foundation, CAREER award CCF-0968667 and an AMD grant.

Authors' addresses: Y. Yang, P. Xiang, and H. Zhou, Electrical and Computer Engineering Department, North Carolina State University, Raleigh, NC; email: yyang14@ncsu.edu; J. Kong and M. Mantor, Graphics Products Group, Advanced Micro Devices.

Permission to make digital or hard copies of part or all of this work for personal or classroom use is granted without fee provided that copies are not made or distributed for profit or commercial advantage and that copies show this notice on the first page or initial screen of a display along with the full citation. Copyrights for components of this work owned by others than ACM must be honored. Abstracting with credit is permitted. To copy otherwise, to republish, to post on servers, to redistribute to lists, or to use any component of this work in other works requires prior specific permission and/or a fee. Permissions may be requested from Publications Dept., ACM, Inc., 2 Penn Plaza, Suite 701, New York, NY 10121-0701 USA, fax +1 (212) 869-0481, or permissions@acm.org.

(C) 2012 ACM 1544-3566/2012/06-ART9 $\$ 10.00$

DOI 10.1145/2207222.2207225 http://doi.acm.org/10.1145/2207222.2207225 
programs remains challenging for application developers. Furthermore, different GPUs (e.g., AMD/ATI GPUs vs. NVIDIA GPUs) may have significantly different hardware features and even the GPUs from the same vendor (e.g., NVIDIA GTX480 vs. GTX285) have quite different characteristics due to the fast evolution of GPU hardware architectures. This fact makes the code developed and tuned for one GPU (e.g., NVIDIA GTX 285) less optimal for a different one (e.g., NVIDIA GTX 480). Our envisioned solution to these problems is to simplify the GPU hardware abstraction model for programmers. Instead of exposing hardware details such as shared memory, thread hierarchy, caches, we propose a GPU hardware abstraction as an array of independent processors connected to the off-chip memory. This way, we encourage application developers to focus on extracting fine-grain thread-level parallelism and/or data-level parallelism from their application algorithms. An optimizing compiler, on the other hand, will perform hardware-specific low-level performance optimizations.

Our compiler works as follows. The input is a naïve GPU kernel function, which is developed based on our simplified GPU hardware abstraction model. The naïve kernel is functionally correct but does not include any device-specific performance optimizations. Such a kernel function represents the user-identified fine-grain work items that can run concurrently. A typical example of a fine-grain work item is the computation of single data element in the output domain and very often it corresponds to single outer-loop iteration in the CPU code. The compiler analyzes the naïve kernel and generates two kernels, one optimized for global memories and the other for texture memories. For the global memory version, the compiler checks the off-chip memory access patterns, and optimizes the memory accesses through vectorization and coalescing to achieve high data access bandwidth. For the texture memory version, the compiler uses aggressive vectorization to exploit the bandwidth provided by texture caches. Then the compiler analyzes data dependencies and identifies possible data sharing across threads and thread blocks. Based on data sharing patterns, the compiler intelligently merges threads and/or thread-blocks to improve memory reuse through the register file, the on-chip shared memory, and the texture cache. These merges provide a novel way to achieve loop tiling and unrolling by aggregating fine-grain work items into threads and thread blocks. Additionally, the compiler schedules the code to enable data prefetching so as to overlap computation with memory access latencies. To avoid partition camping [Ruetsch and Micikevicius 2009] (i.e., to distribute memory traffic evenly across memory partitions), thread blocks are checked for their memory accesses and depending on the thread block dimensions, either an address offset is inserted or the block identifiers (ids) are remapped, if necessary. The compiler also performs hardware-specific tuning based on hardware parameters such as the register file size, the shared memory size, and the number of cores in the target GPU.

Besides the aggressive compiler optimizations, another distinguishing feature of our compiler is that the optimized code is reasonably understandable compared to the code generated using algebraic frameworks such as polyhedral models [Pouchet et al. 2007]. As a result, it is relatively easy to reason about the optimized code generated by our compiler, which facilitates algorithm-level exploration.

In our experiments, we use the compiler to optimize 10 scientific and image processing functions for different GPUs, including NVIDIA GTX 285, NVIDIA GTX 480 and AMD/ATI HD 5870. The experimental results show that our optimized code can achieve very high performance, either superior or very close to the NVIDIA CUBLAS 3.2 library and up to $62 \mathrm{X}$ over the naïve implementation on NVIDIA GTX 285, up to $12 \mathrm{X}$ on NVIDIA GTX 480 and up to $87 \mathrm{X}$ over AMD/ATI HD 5870 . 
In summary, our work makes the following contributions.

(1) We propose a compiler for GPGPU programming that enables the application developers to focus on algorithm-level issues rather than low-level hardware-specific performance optimizations.

(2) We propose a set of new compiler optimization techniques to improve memory access bandwidth, to effectively leverage on-chip memory resource (register file, shared memory, and texture cache) for data sharing, and to eliminate partition conflicts.

(3) Our compiler generates two versions of the code with one optimized for global memory and the other for texture memory. To our knowledge, our compiler is the first to perform code optimizations to automatically utilize texture memory for GPGPU.

(4) We show that the proposed optimizing compiler is highly effective for different GPUs and the programs optimized by our compiler achieve very high performance, often superior to manually optimized codes.

The remainder of the article is organized as follows. In Section 2, we present a brief background on the GPGPU programming model including NVIDIA CUDA [NVIDIA 2010] and OpenCL [OpenCL] and then highlight key requirements for high performance GPU computation. In Section 3, we present our proposed optimizing compiler in detail. Section 4 explores the design space of our propose optimizations. Case studies of matrix multiplication and matrix-vector multiplication are presented in Section 5 to illustrate the compilation process for global memory and texture memory, respectively. The experimental methodology and results are presented in Section 6. In Section 7, we highlight the limitations of the proposed compiler. Related work is discussed in Section 8. Finally, Section 9 concludes our article and discusses future work.

\section{BACKGROUND}

State-of-the-art GPUs employ many-core architectures. The on-chip processors cores are organized in a hierarchical manner. In the NVIDIA GT200/GF100 architecture, a GPU has a number of streaming multiprocessors (SMs) (30 SMs in a GTX 285 and 15 SMs in a GTX 480) and each SM contains multiple streaming processors (SPs) (8 in a GTX 285, 32 in a GTX 480). AMD/ATI HD 5870 GPU has similar architecture and has 20 SIMD engines (i.e., SMs). Each SIMD has 16 stream cores (i.e., SPs). The on-chip memory resources include register files $(64 \mathrm{kB}$ per SM in GTX $285,128 \mathrm{kB}$ per SM in GTX 480 , and $256 \mathrm{kB}$ per SIMD in HD 5870), shared memory (16kB per SM on GTX $285,16 \mathrm{kB}$ or $48 \mathrm{kB}$ on GTX 480 depending on configuration, and $32 \mathrm{kB}$ per SIMD in HD 5870), and caches for different memory regions. To hide the long off-chip memory access latency, these GPUs support a high number of threads to run concurrently. These threads follow the single-program multiple-data (SPMD) program execution model. They are grouped in 32-thread warps/64-thread wavefronts with each warp/wavefront being executed in the single-instruction multiple-data (SIMD) manner. According to the CUDA programming guide [NVIDIA 2010] and ATI Stream SDK OpenCL Programming Guide [AMD 2011], each warp/wavefront contains threads of consecutive, increasing thread ids. In a typical 2D/3D execution domain, the threads in a warp (if not at the boundary) have increasing thread ids along the $X$ direction, and the same thread ids along the $Y$ and $Z$ directions.

In the CUDA/OpenCL programming model, the code to be executed by GPUs is the kernel functions. All the threads (called work items in OpenCL) will run the same kernel code with different thread ids to determine their workloads. The software 
architecture also defines the concept of a thread block/workgroup as an aggregation of threads which must be executed in the same SM and the threads in the same thread block can communicate with each other through the shared memory on the SM.

Next, we summarize the key aspects for high performance GPGPU code as they are the main focus of our proposed compiler optimizations.

(1) Global memory access bandwidth. To utilize the global memory bandwidth efficiently, memory accesses need to be coalesced and each data item may need to be a vector type, depending on specific GPU hardware. Memory coalescing refers to the requirement that the accesses from 16 consecutive threads in a warp (i.e., a half warp) can be coalesced into a single memory access. According to CUDA programming guide [NVIDIA 2010], for devices of compute capability 1.0 and 1.1 (e.g., GTX 8800), the accesses from threads in a half warp must be consecutive and aligned. For devices of compute capability 1.2 and 1.3 (e.g., GTX285), these accesses do not need to be consecutive but still need to be aligned. For devices of compute capability 2.0 (e.g., GTX480), the L1 data cache is used to reduce the requirement on coalescing. AMD/ATI HD5870 has the similar requirement to GTX285. In this article, we use the requirements for coalescing based on compute capability 1.2 and 1.3 and show that the devices of compute capability 2.0 (i.e., GTX480) also benefits from coalescing. We refer to such a coalesced aligned region as a coalesced segment. If each memory access is of the type 'float', each segment starts from an address which is a multiple of 64 bytes, and has the size of 64 bytes. The memory bandwidth utilization may be significantly improved when each of coalesced memory accesses is of a vector data type, such as float2 (a vector of two float numbers) and float4 (a vector of four float numbers). We use a simple copy kernel with one-dimension thread blocks to quantify the impact of vector data types on memory bandwidth. For AMD/ATI HD 5870, the sustained bandwidth reaches $112 \mathrm{~GB} / \mathrm{s}, 148 \mathrm{~GB} / \mathrm{s}$, and $150 \mathrm{~GB} / \mathrm{s}$ when accessing $128 \mathrm{MB}$ data using the float, float2, and float4 data types, respectively. In comparison, for the same data transmission on NVIDIA GTX 285, the sustained bandwidth is $128 \mathrm{~GB} / \mathrm{s}, 133 \mathrm{~GB} / \mathrm{s}$, and $106 \mathrm{~GB} / \mathrm{s}$ using the float, float2, and float4 data types, respectively. On NVIDIA GTX 480, the sustained bandwidth is $145 \mathrm{~GB} / \mathrm{s}, 146 \mathrm{~GB} / \mathrm{s}$, and $145 \mathrm{~GB} / \mathrm{s}$ using the float, float2, and float4 data types, respectively.

(2) Texture memory access bandwidth. Both AMD/ATI and NVIDIA GPUs have onchip caches for texture memory. Our micro benchmarks, in which each thread reads the its texture data multiple times to compensate the effect of cold misses, show the texture cache on HD5870 has higher bandwidth $(1250 \mathrm{~GB} / \mathrm{s})$ than its on-chip shared memory, called local data share $(992 \mathrm{~GB} / \mathrm{s})$. In comparison, GTX 480 has lower bandwidth (336GB/s) than its shared memory (1223GB/s). On GTX 285 the texture cache bandwidth is $310 \mathrm{~GB} / \mathrm{s}$ and shared memory bandwidth is $1011 \mathrm{~GB} / \mathrm{s}$. The advantage of texture memories is that texture memory accesses do not need to be coalesced since texture caches will be checked before accessing the off-chip texture memory. Therefore, the key to performance is to hit in texture caches as often as possible rather than memory coalescing.

(3) Shared memory. The common usage of shared memory is a software-managed cache for memory reuse. Although it has low access latencies, shared memory is slower than register files and has certain overheads beyond access latency. First it needs to be synchronized to ensure proper access order among the threads in a thread block. Second, the shared memory has a number of banks to achieve high bandwidth, and bank conflicts can impair the performance.

(4) Balanced resource usage. As multiple threads in the same thread block and multiple thread blocks compete for limited resources in an SM, including the register file, 


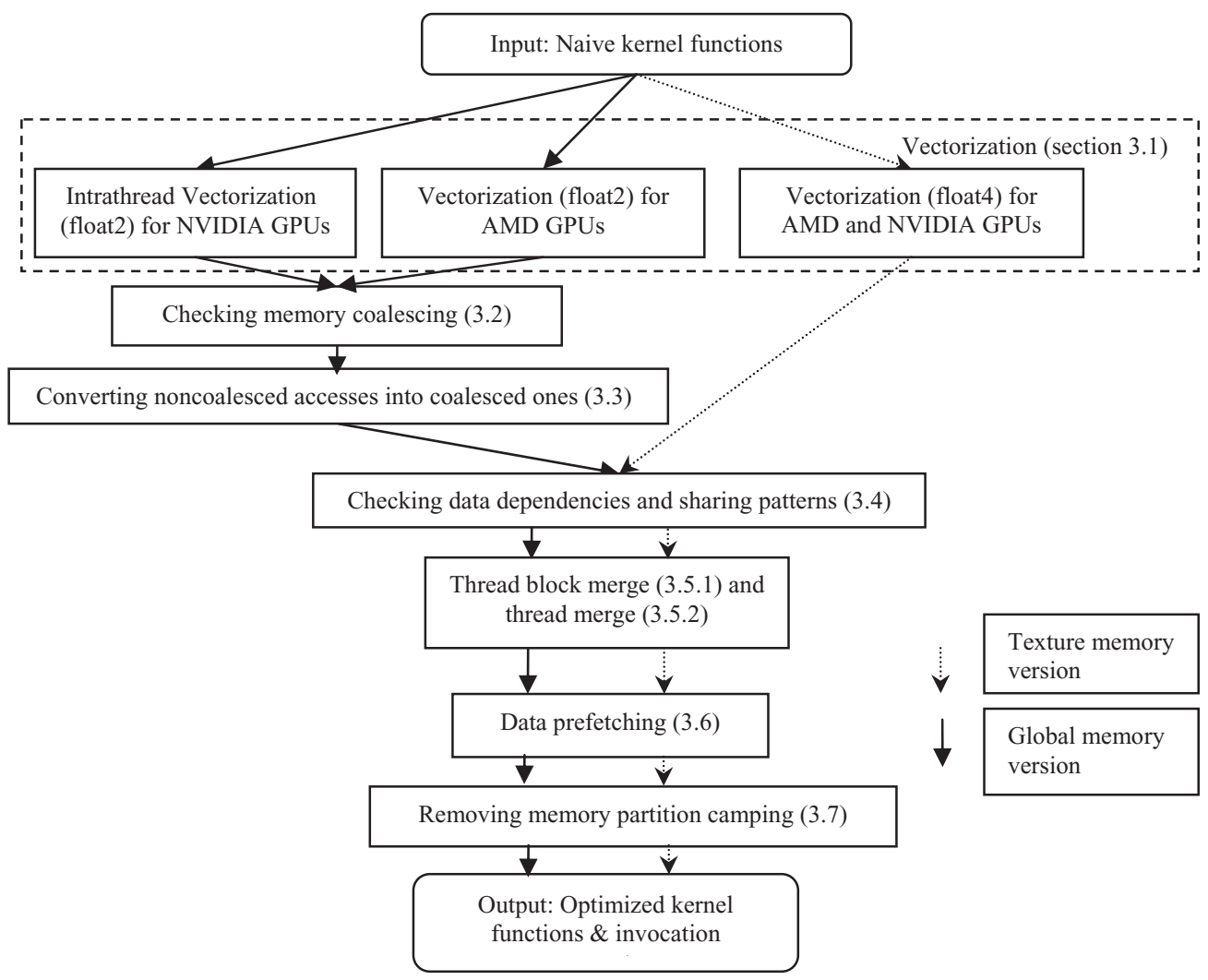

Fig. 1. The framework of the proposed compiler.

the shared memory, and the number of the thread contexts being supported in hardware, we need to carefully balance parallelism and memory optimizations.

(5) Off-chip memory partitions. In current GPUs, off-chip memory is divided into multiple partitions. There are 6 and 8 partitions in NVIDIA GTX 480 and AMD/ATI HD5870/NVIDIA GTX 285, respectively, and the partition width is 256 bytes. To use the partitions effectively, the memory traffic should be evenly distributed among all the partitions. Otherwise, the requests may be queued up at some partitions while others are idle. This is referred to as partition camping [Ruetsch and Micikevicius 2009] or partition conflicts, which are similar to bank conflicts at shared memory but incur much higher performance penalties. Since concurrent memory requests are issued on a per half-warp basis from all active thread blocks, partition conflicts happen across different thread blocks.

Note that the key performance issues just listed are not unique to current GPUs. Future many-core architectures will probably use similar approaches to achieve high memory bandwidth (i.e., coalescing, multiple memory partitions) and to reduce memory access latency (i.e., on-chip cache or shared memory). So, the proposed compiler optimizations are expected to be relevant beyond the scope of GPGPU.

\section{AN OPTIMIZING GPGPU COMPILER}

Our proposed compiler framework is shown in Figure 1. 


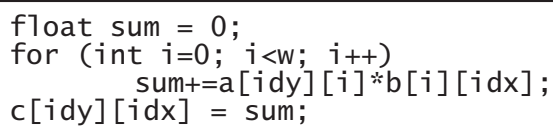

(a) A naïve kernel for matrix multiplication

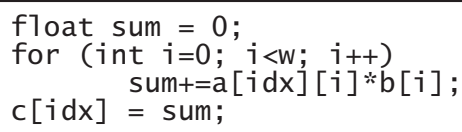

(b) A naïve kernel for matrix-vector multiplication

Fig. 2. Examples of naive kernel functions.

The input to our compiler is a naïve GPU kernel function developed using our simplified GPU hardware abstraction model. The naïve kernel is functionally correct, but does not include any device-specific performance optimizations. For many scientific computing and media processing functions, the naïve version is the code to compute one element/pixel in the output matrix/image. Typically such code is straightforward to extract from the sequential CPU code. One common example is the loop body of a heavily executed loop. In such a case, the subsequent optimization steps (e.g., thread/threadblock merge) have direct correspondence to loop-level optimizations (loop unrolling and titling in particular as discussed in Section 3.5). In Figures 2(a) and 2(b), we show the sample naïve kernel functions for the matrix multiplication $(\mathrm{mm})$ and matrix-vector multiplication (mv) algorithms, respectively. Each computes one element at the position (idx, idy).

In Figure 2, idx and idy are the position/coordinate of the element in the output matrix. In the CUDA/OpenCL programming model, idy can be viewed as the absolute thread id along the Y direction, which is equal to (blockIdx.y*blockDimy + threadIdx.y) in the CUDA code and get_global_id(1) in OpenCL. Correspondingly, idx is the absolute thread id along the $\mathrm{X}$ direction, which equal to (blockIdx.x*blockDimx + threadIdx.x) in CUDA and get_global_id(0) in OpenCL. In comparison, the CUDA predefined threadIdx.x (get_local_id(0) in OpenCL) and threadIdx.y (get_local_id(1) in OpenCL) are the relative thread position/coordinate within a thread block and we refer to them as "tidx" and "tidy" for short. Both tidx and tidy are independent of the thread block ids.

As can be seen from the two examples, the naïve kernel functions don't have any shared memory usage and do not require thread block partition. In other words, we may simply assume every block has only one thread. All the arrays are initially in the off-chip global memory.

For applications which require synchronization among computing different output pixels, For instance, reduction operations, a global sync function is supported in the naïve kernel.

To facilitate compiler optimizations, the following (optional) information can be conveyed using the \#pragma interface: the size of the input and output dimensions, and the output variable names. The latter can be used to eliminate global memory writes to temporary variables when they are moved to shared memory.

Given the naïve kernel function, the compiler generates two kernels, one optimized for global memories and the other for texture memories. For the global memory version, it takes the following steps. First, depending on the targeted GPUs, the compiler attempts to group memory accesses into vector data accesses. Second, the off-chip memory accesses are checked to see whether they satisfy the requirements for memory coalescing. If not, the code will be converted to coalesced memory accesses using shared memory as temporary storage. Third, the compiler analyzes data dependencies and sharing patterns to determine how the data are shared among the neighboring thread blocks. The data reuse information is also used to disable certain memory coalescing transformations when there is little or no data reuse. Based on data sharing patterns, the compiler merges both threads (i.e., combining several threads in different thread blocks into one) to enable the data reuse through registers and thread blocks 


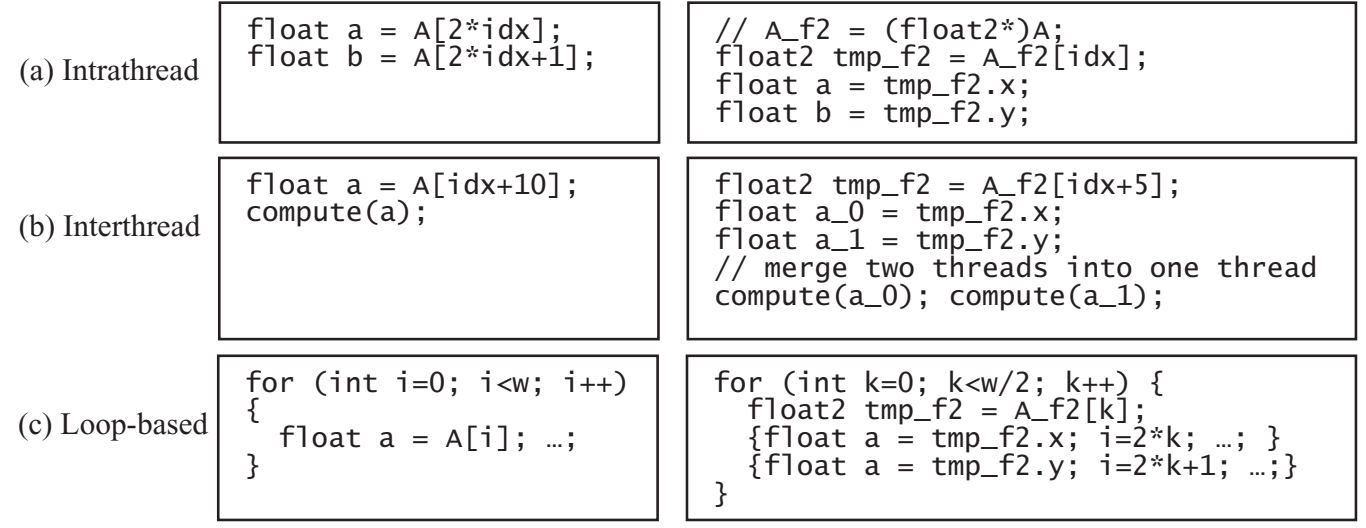

Fig. 3. Three types of vectorization.

(i.e., combining several blocks into one) to increase data reuse through shared memory. After thread/thread-block merge, the compiler schedules the code to perform data prefetching. Then, the compiler checks the memory accesses from different thread blocks for partition camping and either inserts address offsets or remaps thread block ids, if necessary. Finally, the compiler generates one optimized kernel and the parameters (i.e., the thread grid \& block dimensions) to invoke the kernel function.

For the texture memory version, the compiler applies more aggressive vectorization techniques but skips the step for memory coalescing as the texture caches eliminate the need for coalesced memory accesses. As the texture memory version does not utilize the shared memory, the thread-block merge step is simplified compared to the global memory version. The rest of the steps are essentially the same as those for the global memory version, as shown in Figure 1.

The optimization process described above can also be used as a generic methodology to guide manual optimizations of GPGPU programs. As a result, our optimized code is reasonably understandable, as will be seen in the remainder of Section 3.

\subsection{Vectorization of Memory Accesses}

As discussed in Section 2, the data type of memory accesses may have significant impact on bandwidth utilization. Therefore, the compiler first checks data accesses inside the kernel function to see whether they can be grouped in a vector type data access. Since different GPUs favor different vector types, the compiler follows different rules to adjust the aggressiveness of vectorization. We consider the following three types of array accesses for vectorization.

(1) if there is a pair of accesses to the same array in a thread with the indices: $2 * \mathrm{idx}+N$ and $2 * \mathrm{idx}+N+1$, where $N$ is an even number, the compiler generates a float2 variable $\mathrm{f} 2$ with array offset as $\mathrm{idx}+N / 2$ and replaces the original array accesses with $\mathrm{f2} . \mathrm{x}$ and $\mathrm{f2.y.} \mathrm{We} \mathrm{call} \mathrm{this} \mathrm{type} \mathrm{of} \mathrm{vectorization} \mathrm{intra-thread} \mathrm{vectorization,}$ as shown in Figure 3(a). One typical opportunity for intrathread vectorization is complex numbers when the real part is stored next to the imaginary part of each data element.

(2) If there is one global memory access to the index: idx $+N$, where $N$ is an even number, the compiler merges two neighbor threads in the $X$ direction into one thread, generates a float2 variable $\mathrm{f} 2$ with the array offset as $(\mathrm{idx}+N / 2)$ and replaces the original array accesses with $\mathrm{f} 2 \mathrm{x}$ and $\mathrm{f} 2 . \mathrm{y}$. We call this type of vectorization interthread vectorization, as shown in Figure 3(b). 
(3) If there is global memory access to the index: $i+N$, where $N$ is the even number and $i$ is a loop iterator variable with the increment being 1 , the compiler unrolls the loop by 2 , generates a float 2 variable $f 2$ with array offset as $i / 2+N / 2$, and replaces the original array accesses with $\mathrm{f} 2 . \mathrm{x}$ and $\mathrm{f} 2 . \mathrm{y}$. We call this type of vectorization loop-based vectorization, as shown in Figure 3(c).

The target data type of vectorization can be either float2 or float4. Due to the different impacts on bandwidth, different vectorization strategy is used for different memory types (texture vs. global) and different GPUs, as shown in Figure 4. Our proposed vectorization is independent on conditional code in GPU kernel functions. In other words, we attempt to vectorize all the memory access statements no matter whether they are under control of a branch or not. The only limitation is that the statements to be vectorized need to be in the same basic block. For the global memory version on NVIDIA GPUs, a vector of two floats (i.e. float2) is the preferred data type. Considering that the bandwidth improvement of float2 over float is less than 3\%, the compiler only applies intra-thread vectorization. The reasons are (1) inter-thread vectorization merges neighboring threads, reducing thread-level parallelism; and (2) the vectorized data accesses may not be coalesced, and when the compiler converts them into coalesced ones (Section 3.3) through shared memory, there may be bank conflicts in shared memory. For the naive kernels in Figure 2, the code remains the same after vectorization as there is no opportunity for intra-thread vectorization.

For AMD/ATI GPUs, due to the high impact on bandwidth, the compiler applies all three types of vectorization in order to use the float2 type for the global memory version. The reason why float2 is preferred over float 4 is that float 4 reduces the overall number of the threads too much and the performance difference between float2 and float4 is limited. The compiler also checks the number of reads and writes for each array to determine the priority for vectorization. For arrays with a low number of accesses, the compiler only tries to find opportunities for intra-thread vectorization. As the output arrays have a low number of accesses, the compiler only applies intrathread vectorization on them. For example, for the matrix multiplication kernel shown in Figure 2(a), inter-thread and loop-based vectorization are used on input arrays, $a$ and $b$, while only intra-thread vectorization is applied to the output array $c$. as shown in Figure 5(a). Similarly, for matrix-vector multiplication, loop-based vectorization is used on the input array $a$ and input vector $b$, while the accesses to the array $c$ are not vectorized.

For the texture memory version on both AMD/ATI and NVIDIA GPUs, the target vector is float4, because these GPUs' texture units prefer 128 bits to maximize the bandwidth. In fact, the OpenCL specification only defines 128-bit data types (float4, int4 and etc) for the texture memory. Although CUDA supports float and float2 for texture memory, their performance is much lower than float4. The compiler also applies intra-thread vectorization on output arrays.

\subsection{Checking Memory Coalescing}

As discussed in Section 2, GPGPU employs the SPMD model and the threads in a single warp execute the kernel function in the SIMD mode. Therefore, in order to determine whether off-chip memory accesses can be coalesced, we need to compute the addresses of each memory access in the kernel function for different threads. As arrays are the most common data structure in scientific and media processing, we consider four types of array indices and affine transformations of these indices.

(1) Constant index. The constant value is used in an array index, for example, the constant integer 5 in a[idy] $[i+5]$. 


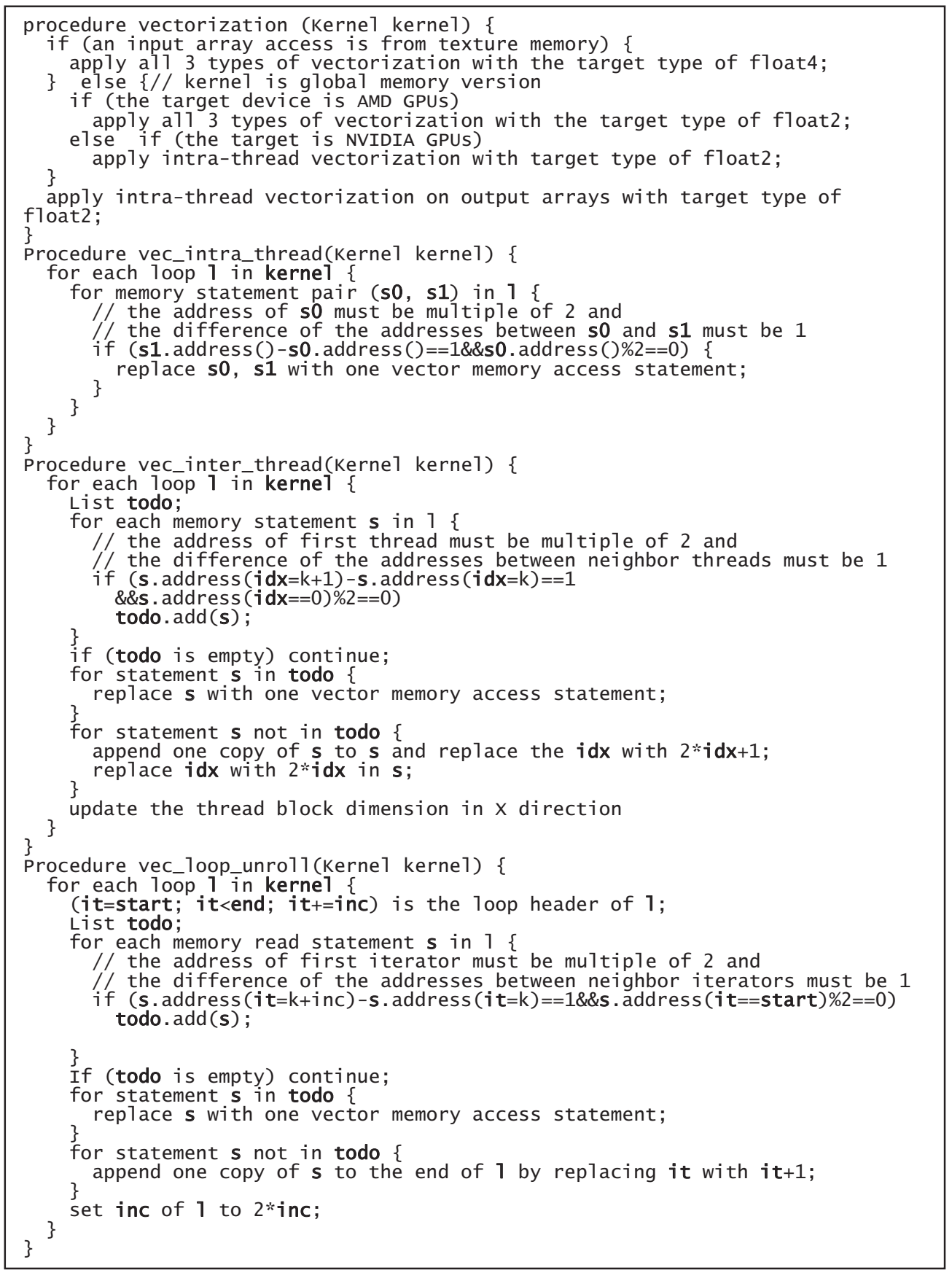

Fig. 4. Pseudocode for vectorization. The procedure "vectorization" shows how to make a decision of the vectorization for different cases. "vec_intra_thread" is the pseudocode for intrathread vectorization, "vec_inter_thread" is for interthread vectorization, and "vec_loop_unroll" is for loop-based vectorization. We only show the float 2 version of vectorization, but the float 4 version is similar. The function "address" calculates the address of the memory access statement using the thread id (idx) and/or loop iterator (it). 


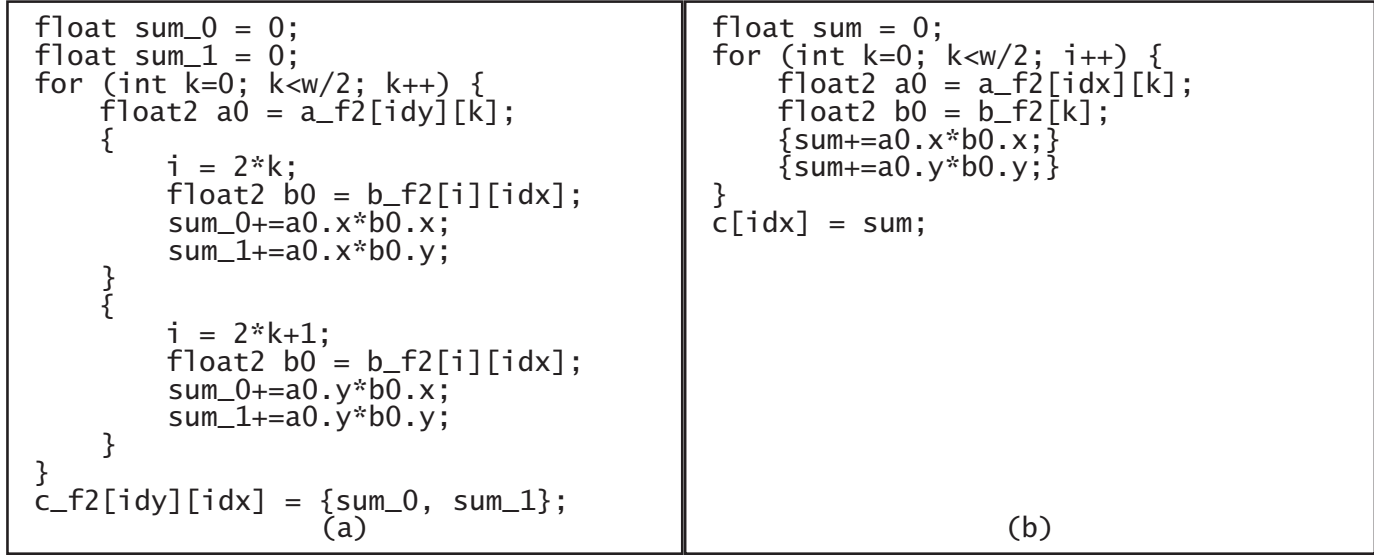

Fig. 5. Examples of vectorization for the global memory version for AMD/ATI GPUs. (a) matrix multiplication after vectorization. (b) matrix-vector multiplication after vectorization.

(2) Predefined index. The predefined numbers, such as absolute thread ids, idx, idy, and relative thread ids, tidx (i.e., threadIdx.x), tidy (i.e., threadIdx.y), are used as an array index. For example, idy in a[idy][i+5].

(3) Loop index. A loop iterator variable is used as an array index, for example, i in b[i] [idx] in Figure 2(a).

(4) Unresolved index. An array index is used, which is not one of the first three types. For example, an indirect access $\mathrm{a}[\mathrm{x}]$ where $\mathrm{x}$ is a value loaded from memory. As our compiler cannot determine the addresses of such indices, we simply skip them without checking whether they can be coalesced.

Among the four types of indices, the addresses corresponding to the first two are fixed for a given thread. For the third, however, we need to check different values of the loop iterator. Assuming that a loop index starts from S with increment Incr, then we need to check the index addresses from the first 16 iterations: $\mathrm{S}, \mathrm{S}+\mathrm{Incr}, \mathrm{S}+2^{*} \mathrm{Incr}$, to $\mathrm{S}+15^{*}$ Incr. The reason is that the same behavior repeats for remaining iterations in terms of whether the access can be coalesced as the difference in addresses is a multiple of 16.

After determining the types of array indices in the kernel function, for each memory access instruction, the compiler computes the addresses from the 16 consecutive threads in the same warp (i.e., a half warp) to see whether they can be coalesced. As discussed in Section 2, if we assume the array type of float, the coalesced accesses will form a coalesced segment, which starts from an address, whose value is a multiple of 64 , and has the size of 64 bytes. Among the addresses from the 16 threads, we refer to the smallest one as the base address. The differences between the base address and the addresses from the subsequent 15 threads are referred to as offsets. To satisfy the coalescing requirement, the base address needs to be a multiple of 64 and offsets need to be 1 to 15 words. The following two rules are used to handle common array accesses.

(1) For an index to a multidimensional array, e.g., $\mathrm{A}[\mathrm{z}][\mathrm{y}][\mathrm{x}]$, the index to the higherorder dimensions, For instance, the y and $\mathrm{z}$ dimensions, should remain the same for all the 16 threads in the half warp. Otherwise, for example, if the predefined index idx (the thread id along the $\mathrm{x}$ direction) is used in an index to the $\mathrm{y}$ dimension in a multi-dimension array $\mathrm{A}[][\mathrm{idx}][0]$, the accesses from the 16 threads will be $\mathrm{A}[][0][0], \mathrm{A}[][1][0], \mathrm{A}[][2][0]$, etc., and are not coalesced. 
(2) When a loop index is used in the kernel function, the compiler computes the base address and the offsets for each possible value of the loop iterator. For example, for the address a[idy][i] in Figure 2(a), the base address is \&a[idy][0] when the iterator $i$ is $0 ; \& a[i d y][1]$ when $i$ is 1 , etc. The offsets are all zeros as the addresses do not change for different threads in the same half warp. As both the base addresses and the offsets do not meet the condition, the array access a[idy] [i] is not coalesced. For the array access b[i][idx] in Figure 2a, the base address is \&b[0][0] when $\mathrm{i}$ is 0 ; $\& b[1][0]$ when $i$ is 1 , etc. The offsets are from 1 word to 15 words. Thus, the array access $b[i][i d x]$ is coalesced as long as each row of array $b$ is aligned to the multiple of 16 words. For the array access b[idx+i], although the offsets satisfy the condition for every possible $i$, it is not a coalesced access since the base address is not always a multiple of 16 words, for instance, b[1] when $i$ is 1 .

\subsection{Converting Noncoalesced Access into Coalesced Ones}

After the compiler analyzes every array access in the kernel code, the compiler converts the noncoalesced global memory accesses into coalesced ones through shared memory. The observation here is that for each noncoalesced memory access instruction, the compiler can determine the coalesced segments that contain the data required by the noncoalesced memory accesses from the half warp. The compiler then introduces shared-memory array variables, inserts statements (coalesced memory accesses) to initialize the shared memory variables, and replaces the original global memory accesses with shared memory accesses. The thread block size is also set to 16 so that each thread block contains one half-warp. The syncthreads function is also inserted to ensure the proper access order.

For array accesses using constant or predefined indices, the process is typically straightforward. For example, for the noncoalesced access, A[idy][0], the coalesced segment is $\mathrm{A}[\mathrm{idy}][0: 15]$. The compiler inserts a shared-memory array variable $\mathrm{sA}[0: 15]$ and initializes the $\mathrm{sA}[0: 15]$ with $\mathrm{A}[\mathrm{idy}][\mathrm{tidx}]$, where tidx is thread relative id within the warp. In the case when idx is used in an index to a multi-dimensional array, the compiler may introduce a loop to load the required data for a half warp. For example, for an array access $\mathrm{A}[\mathrm{idx}][0]$, the required data for a half warp is $\mathrm{A}[(\mathrm{idx}-\mathrm{tidx})+(0: 15)][0]$, where (idxtidx) provides the start address of each thread block, which is the same as the start address of the half warp as each thread block only contains a half warp at this time. The coalesced segments that contains the required data are $\mathrm{A}[(\mathrm{idx}-\mathrm{tidx})+(0: 15)][0: 15]$. In the introduced loop of 16 iterations, a shared memory array is initialized with $\mathrm{A}[(\mathrm{idx}-$ tidx $)+1][t i d x]$, where $l$ is the iterator of the newly introduced loop. From these examples, it can be seen that not all the data loaded in the shared memory are useful, the compiler will perform data reuse analysis (Section 3.4) to determine whether this transformation is beneficial or not. If it is not, the compiler will skip coalescing transformation on this access. In the special case where an array access involves both idx and idy, such as $\mathrm{A}[\mathrm{idx}][\mathrm{idy}]$, the compiler analyzes the feasibility to exchange idx and idy to make it coalesced. This transformation is equivalent to loop interchange on the CPU code.

For array accesses using a loop index, $\mathrm{A}[\mathrm{m} * \mathrm{i}+\mathrm{n}]$, where $\mathrm{i}$ is the loop iterator and $m$ and $n$ are constants, the compiler unrolls the loop for $16 /(\mathrm{GCD}(\mathrm{m}, 16))$ times if $m$ is less than or equal to 8 . If $m$ is greater than 8 , the coalesced access has little benefit due to limited reuse across different iterations. Then, the compiler groups the accesses from unrolled loops into coalesced ones. For example, for the array access A[idy][i] where i is the loop iterator, the segment $\mathrm{A}[\mathrm{idy}][0: 15]$ contains all the required data for the first 16 iterations. The compiler unrolls the loop for 16 times, introduces shared memory variable $\mathrm{sA}[0: 15]$ which are initialized with $\mathrm{A}[\mathrm{idy}][\mathrm{tidx}+\mathrm{i}]$ (coalesced as the increment of $i$ is 16 after unrolling), and replaces A[idy][i] with sA[i]. 


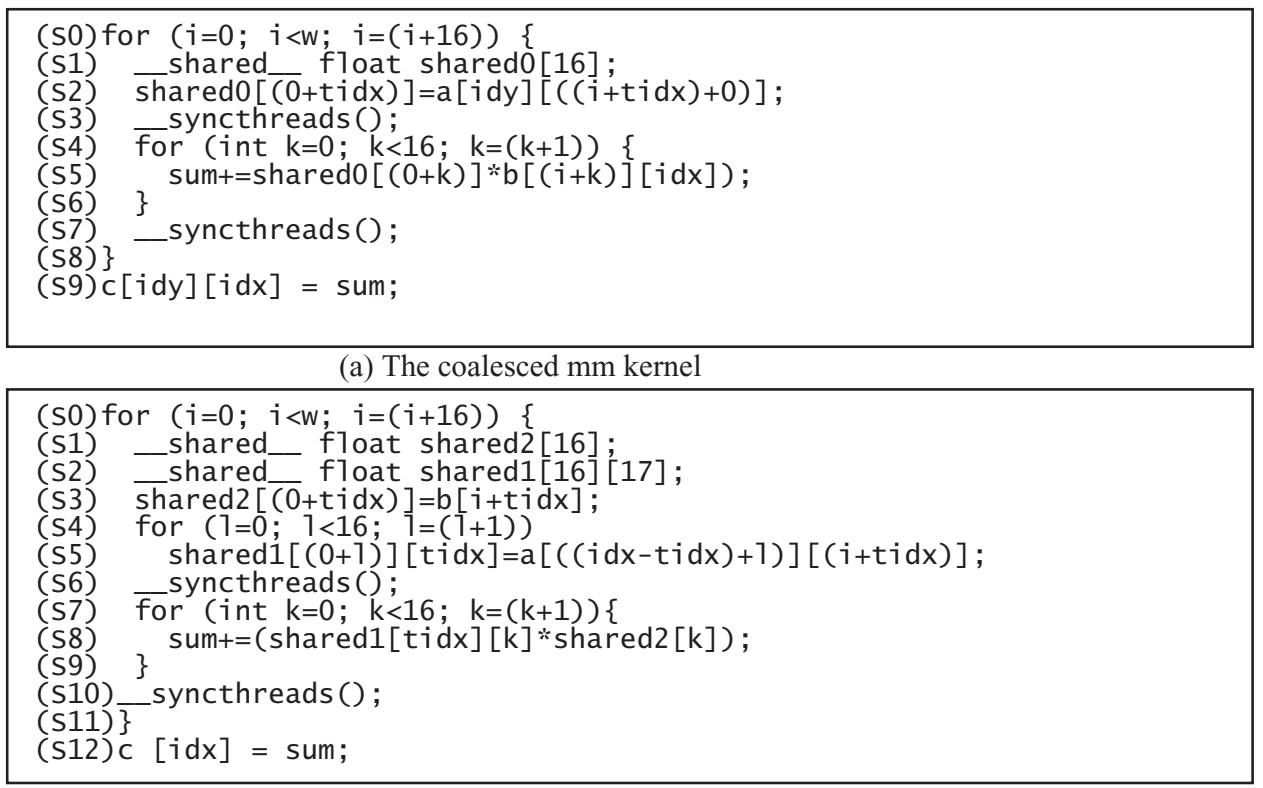

(b) The coalesced mv kernel

Fig. 6. Coalesced kernels generated by the compiler.

For the naïve kernels in Figure 2, the coalesced versions on NVIDIA GPUs are shown in Figure 6. The inner loop with the iterator $\mathrm{k}$ is a result of unrolling the outer loop with the iterator i. In the naïve kernel in Figure 2(a), the access a[idy][i] is not coalesced, which results in loop unrolling as described above. b[i] [idx] is coalesced and it transforms to $\mathrm{b}[(\mathrm{i}+\mathrm{k})][\mathrm{idx}]$ due to unrolling for a[idy][i]. In the mv kernel in Figure 2(b), both accesses a[idx][i] and b[i] are not coalesced. Converting the access $b[i]$ into coalesced accesses involves a loop unrolling of $16(=16 / \mathrm{GCD}(1,16))$ times and it becomes b[i+tidx] in Figure 6(b). For the access a[idx][i] the loop with the iterator $\mathrm{l}$ is introduced and the access is transformed to a[(idx-tidx $)+1][i+t i d x]$. In addition, the compiler will add padding to the shared memory arrays to avoid bank conflicts if necessary. The compiler also assumes that the row size of each global memory array is a multiple of 16 words so as to meet the requirement of memory coalescing. Therefore the programmers may need to add padding to global memory arrays. It is similar to the unroll pragma provided by NVIDIA and AMD compilers, which require the programmers to guarantee that the loop counter is the multiple of the unroll factor.

After memory coalescing, the kernel code generated by our compiler has the following characteristics.

(1) Each thread block has 16 consecutive threads (i.e., only a half warp) along the $\mathrm{X}$ direction, because 16 threads are needed by hardware to coalesce memory accesses and they communicate with each other through shared memory. The number of threads in each thread block will be expanded during the next optimization phase (Section 3.5) to make sure there are enough threads in each thread block.

(2) There are two types of global memory load statements: (a) Global memory to shared memory (G2S): the statements read data from global memory and store them into the shared memory, such as (S2) in Figure 6(a). (b) Global memory to register (G2R): the statements read data from global memory and save them to registers. 
For example, in (S5) in Figure 6(a), the global memory access 'b[(i+k)[idx]' loads the data into registers.

On AMD/ATI GPUs, the compiler will apply vectorization on the global memory first. Therefore the data unit for memory coalescing may be float2 instead of float.

For the texture memory version on both AMD/ATI and NVIDIA GPUs, since texture memory accesses do not need to be coalesced due to texture caches, the compiler skips the steps for memory coalescing.

\subsection{Data Dependencies and Data Sharing}

In this step, the compiler detects data dependency and data sharing. Such analysis is similar to those used in analyzing affine array accesses for locality optimization and parallelization [Aho et al. 1986]. For the global memory version, as our compiler has already enforced memory coalescing by associating coalesced segments with each global memory access, the compiler can detect data sharing by comparing whether the address ranges of the segments have overlaps. For the texture memory version, data sharing is achieved through the register file and texture caches. In the applications that we studied, we found that data sharing happens most frequently among neighboring blocks along the $X$ or $Y$ direction. Therefore, our current compiler implementation mainly focuses on checking data sharing among neighboring thread blocks and also the thread blocks with a fixed stride along the $X$ or $Y$ direction.

The data sharing/reuse information is also used to determine whether the code conversion for memory coalescing is beneficial. As described in Section 3.3, shared memory is used as temporary storage to achieve memory coalescing. The data in the shared memory, however, may not be useful as they are simply loaded from off-chip memory to satisfy the coalescing requirement. For example, the compiler loads A[idy][0:15] in order to convert the access $\mathrm{A}[\mathrm{idy}][0]$ into a coalesced one. Currently, our complier employs a simple rule to check whether an access needs to be converted: if the loaded data in shared memory have no reuse, it is not converted. A more crafted heuristic may further rank code conversions for different accesses by comparing their shared memory usage and number of data reuses, and then select the most beneficial ones if shared memory is used up. We left such investigation as our future work to refine our compiler framework.

\subsection{Thread/Thread-Block Merge to Enhance Memory Reuse}

After detecting that there exists data sharing among thread blocks (mainly neighboring blocks), we propose two new techniques to enhance data sharing so as to reduce the number of memory accesses: merging thread blocks and merging threads. Thread-block merge determines the workload for each thread block while thread merge decides the workload for each thread. These two techniques combined are essentially a way to achieve loop tiling and unrolling by aggregating the fine-grain work items into threads and thread blocks. We first present the two techniques and then discuss how compiler prioritizes one over the other.

3.5.1. Thread-Block Merge. For the global memory version, when our compiler determines that multiple thread blocks share some common data, it may choose to merge them into one thread block, as shown in Figure 7.

To illustrate the procedure to merge thread blocks, we show how our compiler combines two neighboring blocks along the $X$ direction into one. First, the compiler recomputes the thread id information within the thread block (i.e., tid). As two thread blocks along the $X$ direction are merged, idx, idy and tidy remain the same while tidx is recomputed as (idx\%(N*blockDim.x)), where $N$ is 2 for Figure 7 . Second, for the statements that result in data sharing, we add control flow to ensure that the global memory data 


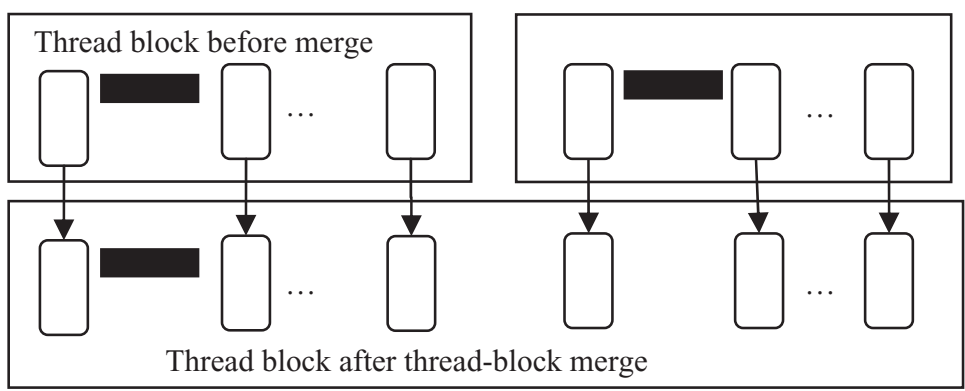

Shared data in shared memory

Fig. 7. Improve memory reuse by merging neighboring thread blocks.

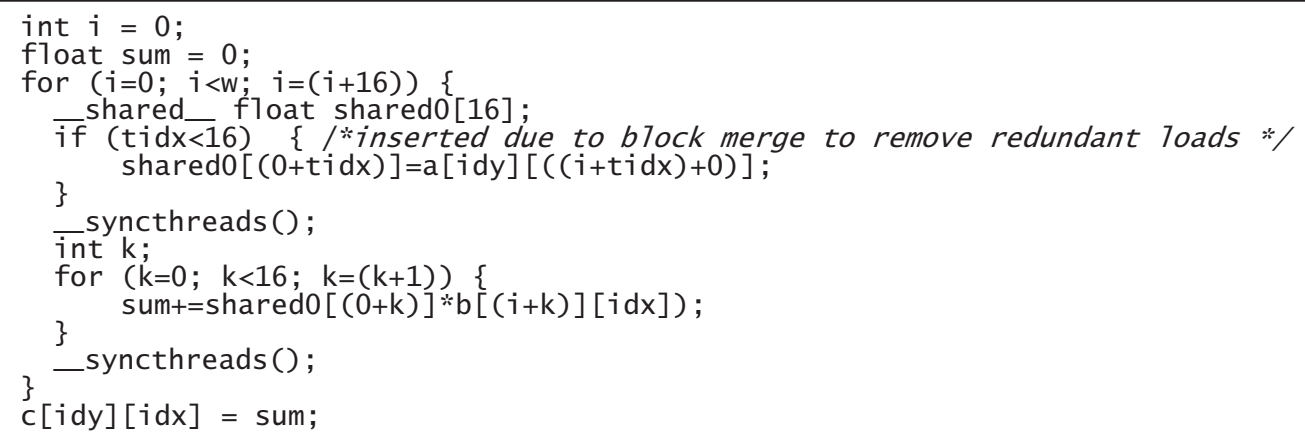

Fig. 8. The kernel function for matrix multiplication, after merging blocks along the $\mathrm{X}$ direction.

are loaded only once. For the matrix multiplication example in Figure 6(a), the statement S2 in threads from two neighboring thread blocks accesses the same segment. Therefore, we add an 'if (tidx < blockDim.x)' statement to eliminate redundant global memory accesses, as shown in Figure 8. Third, the thread block dimension is resized (blockDim.x $=2 *$ blockDim.x).

As thread-block merge determines the workload for each thread block and all threads in the same thread block reuse data in shared memory, it essentially achieves loop tiling for locality and parallelism optimizations.

For the texture memory versions, since they rely on the texture caches rather than shared memory for data reuse, the block merge does not change the kernel code. Instead, the compiler simply adjusts the thread block configuration to exploit data locality. We propose the following rules for thread block configuration: (a) the number of threads in a thread block is set as the warp/wavefront size and the initial set up of the thread block configuration is $32 \times 1$ (64 $\times 1$ for a wavefront) for one-dimension output domain and $8 \times 4$ ( $8 \times 8$ for a wavefront) for two-dimension output domain; (b) if the threads in the same warp/wavefront exhibit spatial locality along the $X$ direction, e.g., an array access with the index $\mathrm{A}[][][\mathrm{idx}]$, the block dimension on the $X$ direction is set to be at least $k$, where $k$ is the ratio of the texture cache line size over the size of the datum to be accessed. If the data type is float 4 and the texture cache line size is 64 Bytes, the ratio is 4 , implying that there are at least 4 threads in a warp/wavefront can reuse a cache line in texture caches; and (c) similarly, if the threads in the same warp/wavefront exhibit spatial locality along the $Y$ direction, e.g., an array access with the index A[][][idy]., the block dimension on the $Y$ direction is set to be at least $k$ as well. 


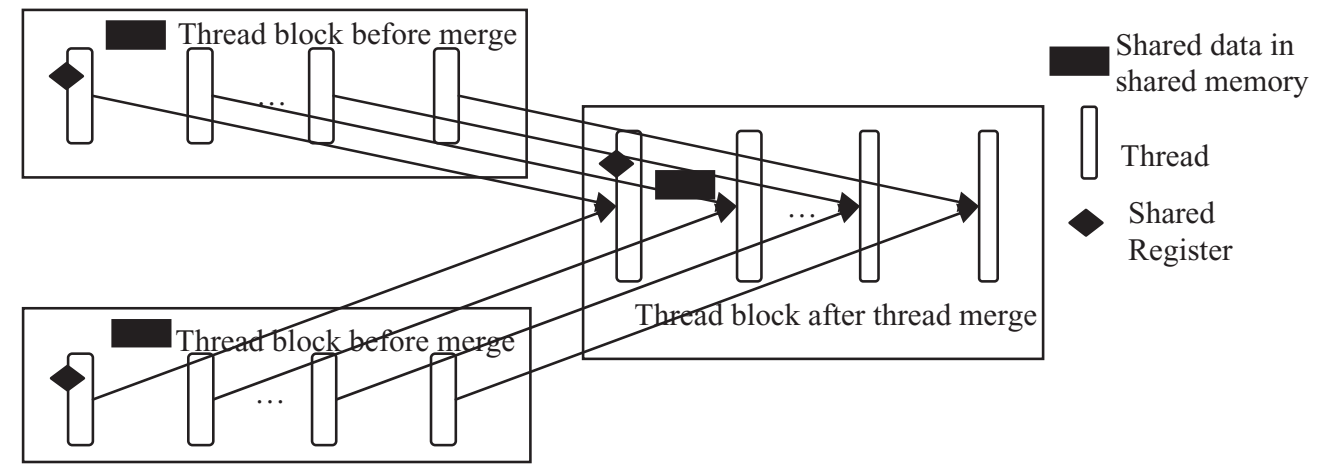

Fig. 9. Improve memory reuse by merging threads from neighboring thread blocks.

3.5.2. Thread Merge. The other approach to enhance data sharing is to merge threads from different thread blocks, which combines several threads' workloads into one, as shown in Figure 9. Compared to thread-block merge, after these threads are combined into one, they can share not only shared memory, but also the registers in the register file. Furthermore, some control flow statements and address computation can be reused, thereby further reducing the overall instruction count. The limitation is that an increased workload typically requires a higher number of registers, which may reduce the number of active threads that can fit in the hardware. From the discussion, it can be seen that thread merge achieves the effects of loop unrolling. Note that thread merge also combines multiple thread blocks into one but it does not increase the number of threads in each thread block. Thread merge is the same for both the global memory version and the texture memory version.

To illustrate the procedure to merge threads, we show how our compiler combines $N$ neighboring blocks along the $Y$ direction into one. First, the compiler recomputes the thread id information. As we merge threads from two thread blocks along the $Y$ direction, the absolute thread ID along the $X$ direction idx remains the same while the thread ID along the $Y$ direction idy will be changed to idy* $\mathrm{N}$, idy* $\mathrm{N}+1$, idy* $\mathrm{N}+2 .$. , idy* $\mathrm{N}+(\mathrm{N}-1)$ for the $N$ replicated statements. The thread id information within a thread block remains the same. Second, for the statement that results in data sharing, we need only one copy. Third, for the control flow statement such as loops, we also only need one copy. Fourth, for the remaining statements including data declaration, ALU computation statement and other memory access statements, we replicate them for $N$ times. For the matrix multiplication example in Figure 8, the array access $b[(i+k)][i d x]$ results in the shared data among the thread blocks along the $Y$ direction (as the access address is not dependent on 'idy'). The compiler merges 32 neighboring blocks along the $Y$ direction using thread merge, as shown in Figure 10.

3.5.3. Selection between Thread Merge and Thread-Block Merge. As discussed in Section 3.3, for the global memory version, the code generated by the compiler after memory coalescing has two types of global memory accesses: global to shared memory (G2S) and global to register (G2R). If data sharing among neighboring blocks is due to a G2S access, the compiler prefers thread-block merge to better utilize the shared memory. When data sharing is from a G2R access, the compiler prefers to merge threads from neighboring blocks due to the reuse of registers. If there are many G2R accesses, which lead to data sharing among different thread blocks, the register file is not large enough to hold all of the reused data. In this case, thread block merge is used and shared memory variables are introduced to hold the shared data. In 


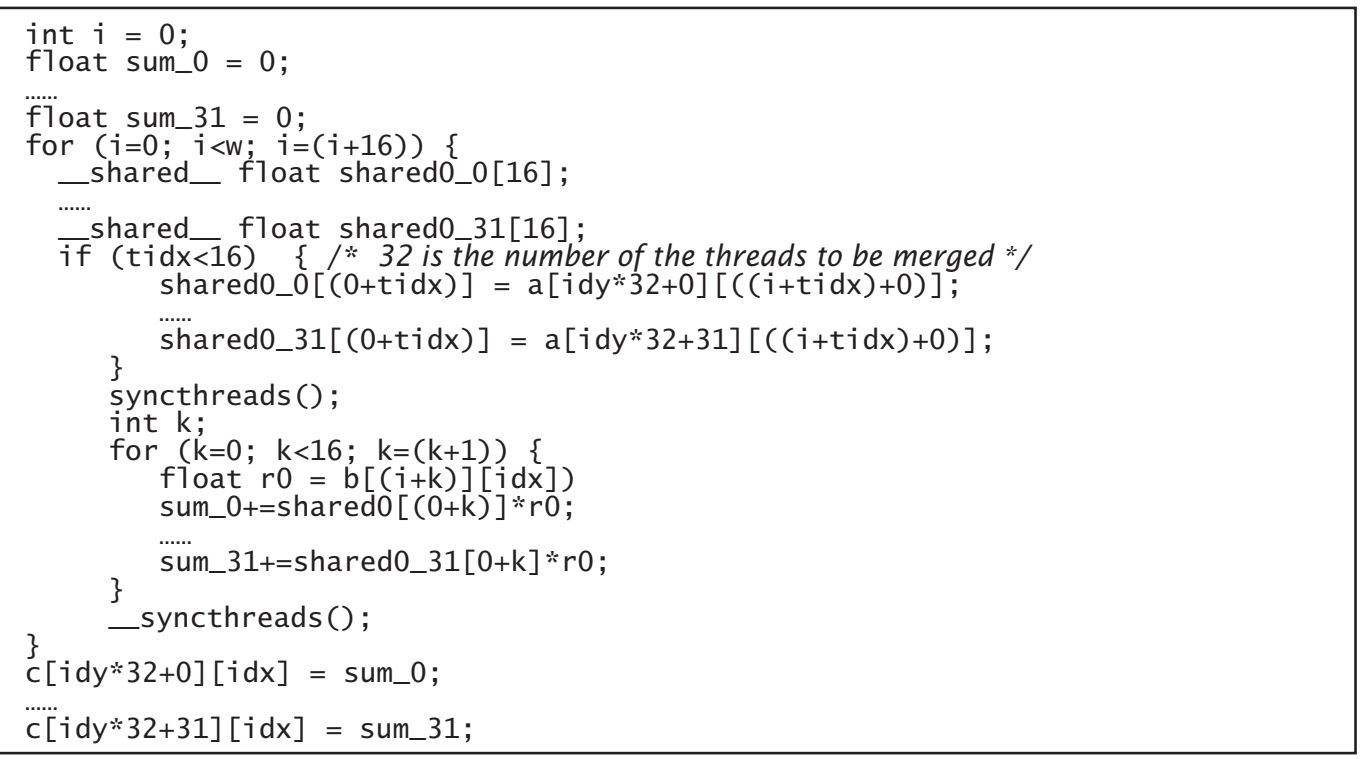

Fig. 10. The matrix multiplication kernel after merging 32 threads in 32 adjacent blocks along the $\mathrm{Y}$ direction.

addition, if a block does not have enough threads, thread-block merge instead of thread merge is also used to increase the number of threads in a block even if there is no data sharing.

Given the different sizes of register file and shared memory on different GPUs, the compiler applies different thread (block) merge policies based on their hardware specifications. On GTX 285, the compiler applies thread block merge along the $X$ or $Y$ direction depending on the data sharing pattern (if there exists data sharing along both directions, the $Y$ direction is selected as the memory coalescing step already combine 16 threads along the $X$ direction into one thread block) followed with thread merge along the $X$ or $Y$ direction. With more resources available on GTX 480 or HD5870, the compiler applies thread block merge along both the $X$ and $Y$ directions followed with thread merge along the $X$ and $Y$ directions. If the compiler cannot find the data sharing in one direction, the merge step along this direction is skipped.

For the texture memory version, all data accesses are from memory to registers. So, thread merge is performed first to exploit reuse along neighboring threads. Then, thread block merge adjusts thread block dimensions to leverage texture caches.

Our compiler also assumes that there is enough thread level parallelism, which means there are enough threads to utilize the GPUs. If the input problem size is small, for example, for 16 by 16 matrix multiplication, thread merge doesn't give any benefit because of the lack of insufficient threads. We leave it to the programmers to choose from naive or optimized kernels depending on their input sizes.

\subsection{Data Prefetching}

Data prefetching is a well-known technique to overlap memory access latency with computation for inner thread. The overhead of data prefetching code is the increased register usage due to the temporary variables. Because the GPUs can overlap memory access latency with computation by using thread level parallelism. Even compiler can support data prefetching, it doesn't give much benefit. 


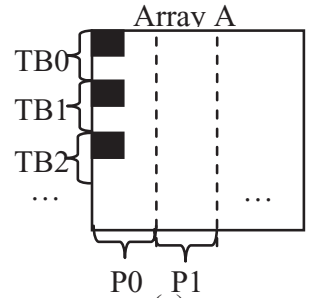

(a)

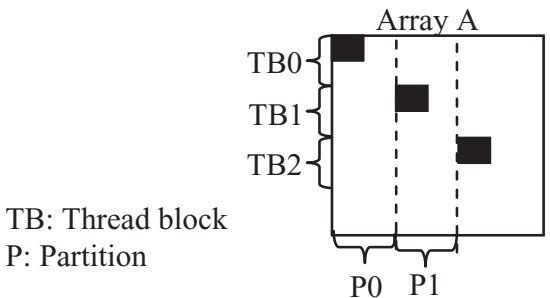

(b)

Fig. 11. Eliminating partition camping. (a) Accesses to array $A$ resulting in conflicts at partition 0 . (b) Adding an offset as (partition size * bidx) eliminates the conflicts. The dark regions represent the memory footprint of $\mathrm{A}[\mathrm{idx}][0]$ from different thread blocks.

\subsection{Eliminating Partition Camping}

In this step, the compiler reuses the address access patterns obtained for thread/threadblock merge to see whether they lead to partition camping. As neighboring thread blocks along the $X$ direction are likely to be active at the same time, the compiler focuses on the addresses that involve blockIdx.x or bidx in short. Those accesses without involving bidx either access the same line in the same partition (e.g., A[0]) or access the same partition at different times (e.g., A[bidy] [0] based on the assumption that thread blocks with different bidy will execute at different times). The following rules are followed by our compiler.

Partition Camping Detection. If an array access involves bidx, the compiler checks the address stride between the two accesses from the two neighboring blocks (i.e., one with block id bidx and the other with bidx +1 ). The compiler detects partition camping if the stride is a multiple of (partition size * number of partitions). For example, for an array access $\mathrm{A}[\mathrm{idx}]$, it is equivalent to $\mathrm{A}[\mathrm{bidx}$ *blockDimx+tidx]. The stride between two neighboring blocks is blockDimx, whose value then decides whether there are partition conflicts (i.e., two concurrent accesses to the same partition).

Partition Camping Elimination. If an access results in partition conflicts, depending on how thread blocks are organized, we use two ways to eliminate partition conflicts.

(1) If thread blocks are arranged in one dimension, we add a fixed offset, (the partition width * bidx), to the access and update the loop bounds to accommodate the change. For example, in mv, the output is a vector. So the thread blocks are organized in one dimension. The accesses $\mathrm{A}[\mathrm{idx}][\mathrm{i}]$ (or the coalesced version $\mathrm{A}[((\mathrm{idx}-$ tidx $)+1)][(i+$ tidx $)]$ ), from neighboring thread blocks result in partition camping if the width of $A$ is a multiple of (partition size * number of partitions), as shown in Figure 11(a). With the added offset, the access pattern is changed to Figure 11(b), eliminating partition camping.

(2) If thread blocks are organized in two or more dimensions, we apply the diagonal block reordering proposed in [Ruetsch and Micikevicius 2009], which essentially changes the workload (or tile) that each thread block is assigned to. The diagonal mapping rule is newbidy $=$ bidx and newbidx $=($ bidx+bidy $) \%$ gridDim. $x$.

\section{DESIGN SPACE EXPLORATION}

\subsection{The Number of Threads in a Thread Block}

In our compiler algorithm, for the global memory version, the number of threads in a thread block is determined by thread/thread-block merge. To better illustrate how the compiler applies thread (block) merge, we show the pseudo code in Figure 12. The basic functions at Lines 1 and 2 are thread block merge and thread merge discussed in 


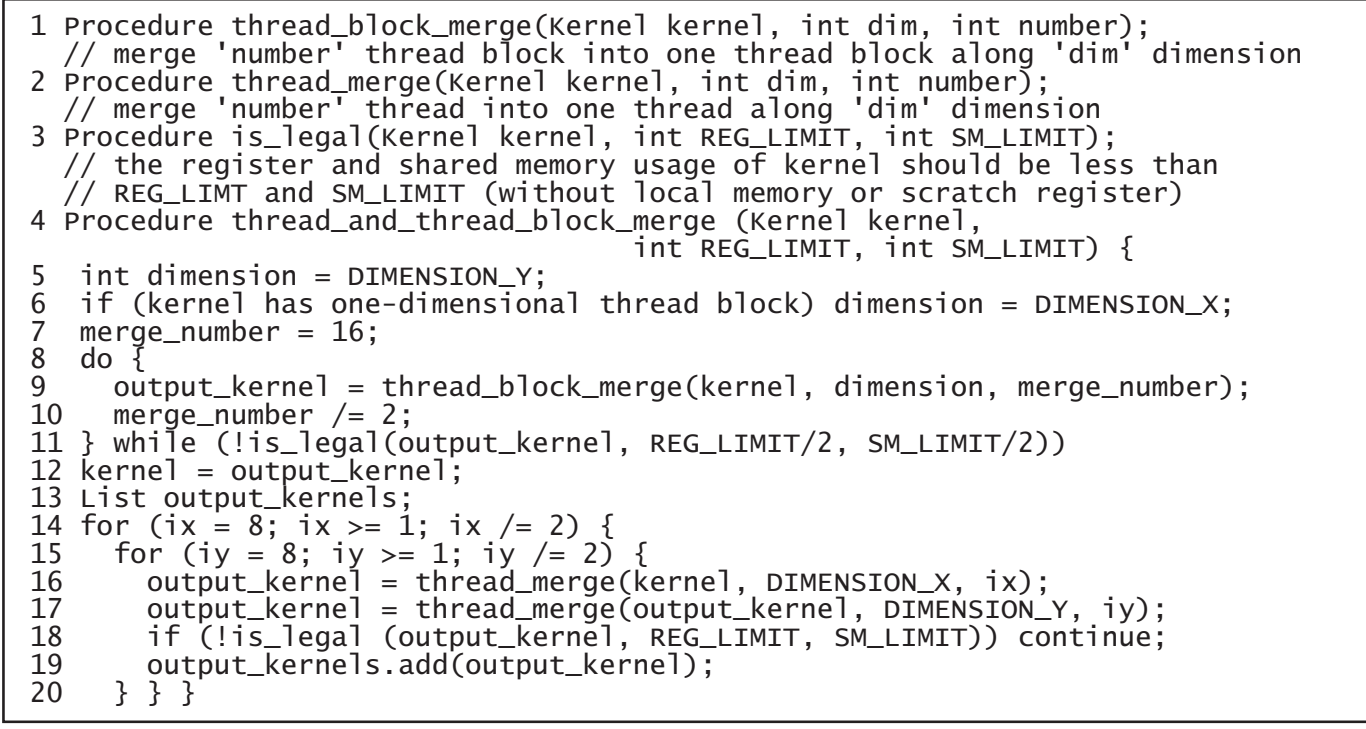

Fig. 12. Algorithm of choosing parameters for thread (block) merge.

Section 3.5. Line 4 is the main function for choosing the parameters for thread and thread block merge. The code between Line 5 and Line 11 is for thread-block merge. The CUDA programming guide suggests that one SM should have at least 192 active threads to hide the latency of register read-after-write dependencies. Because our compiler tries to use a number of resources (the shared memory due to thread-block merge and the register file due to thread merge) for better memory reuse, it is possible that the code after thread/thread-block merge requires a large amount of shared memory and registers so that one SM can only support a limited number of thread blocks. To balance the thread-level parallelism and memory reuse, our compiler tries to put 256 threads into one thread block (equivalent to merging of 16 blocks as Line 7), if possible. Because after coalesced step, there are already 16 threads along the $\mathrm{X}$ dimension, we prefer to perform thread block merge along Y direction as shown in Line 5. However if the thread block has only one dimension, we can only apply thread block merge along $\mathrm{X}$ direction (Line 6). The compiler will also verify the resource usage to make sure that each SM/SIMD can run at least two thread blocks (Line 11). The code between Line 12 and Line 20 is for thread merge. The compiler varies the degrees of thread merge (i.e., how many threads to be merged into one) across 8, 4, 2, 1 along the $\mathrm{X}$ direction (Line 14) and 8, 4, 2, 1 along the Y direction (Line 15) so as to balance register-based data reuse and thread-level parallelism. As such, the combination of these design parameters creates a design space to explore. As discussed in Section 3.5, merging threads/thread blocks is one way to achieve loop tiling and unrolling. So, exploring such a design space is similar to finding the best tile size and unrolling factors for parallelization and locality enhancement. Due to the nonlinear performance effect of those parameters on GPU performance, the compiler generates multiple versions of code and resorts to an empirical search by test running each version to select the one with the best performance. Although the compiler can generate up to 16 versions, typically many of them will be discarded due to the resource usage check at Line 18. Another way is to use an analytical performance model [Hong and Kim 2009; Baghsorkhi et al. 2010] to predict the performance of each version, but this requires higher accuracy than current models. Moreover, based on our experiments, the optimal version may be dependent upon 
Table I. Machine Description

\begin{tabular}{|l|l|}
\hline Parameter & \multicolumn{1}{|c|}{ Description } \\
\hline CoalescedThread & Number of threads required for coalesced memory accesses \\
\hline ThreadInWarp & Number of threads in one warp or wavefront \\
\hline WarpInMP & Maximum number of warps in one MP or wavefronts in one SIMD \\
\hline ThreadInMP & Maximum number of threads in one MP or SIMD \\
\hline ThreadBlockInMP & Maximum number of thread blocks in one MP or work groups in one SIMD \\
\hline RegisterInMP & Number of 32-bit registers in one MP or SIMD \\
\hline ThreadInBlock & Maximum number of threads in one thread block or work group \\
\hline ShareMemoryInMP & Size (KB) of shared memory in one MP or local data shared in one SIMD \\
\hline Vectorization & Targeted vector type on the global memory version \\
\hline CacheLineSize & Size (Bytes) of the texture cache line \\
\hline
\end{tabular}

the size of the input arrays, which implies that unless the compiler knows detailed information of the intended inputs, it is almost inevitable that the compiler must run multiple versions of code in order to find the optimal one.

Both the NVIDIA and AMD compilers limit the number of registers for single thread. For example, if one thread requires more than 64 32-bits registers, the NVIDIA compiler will only allocate 64 registers per thread and spill data into local memory (which is scratch register in AMD GPUs) so as to guarantee as least 512 threads in GTX 480. The AMD compiler also limits 62 128-bits registers per thread to guarantee 256 threads in HD 5870, unless the programmers specify more registers explicitly [AMD 2011]. Our compiler follows the default configurations of the vendors' compilers to ensure there is enough thread level parallelism for each MP or SIMD. Our compiler parses the register usage from the compilation results of the vendors' compilers and make sure the thread merge would not introduce the local memory or scratch register usage, which is the is_legal function at Line 3 of Figure 12. If our compiler detects the local memory or scratch register over-usage, the compiler will discard such kernels as shown at Line 11 in Figure 12.

\subsection{Hardware Specification}

GPU hardware is evolving rapidly. Although different generations of GPU hardware may share similar architecture, for instance, NVIDIA GTX 285, GTX 480 and AMD/ATI HD 5870, there are significant variations, e.g., the size of the register files, the size of the shared memory, which may have a strong impact on performance. As a result, an optimized code tuned for one GPU generation may not be optimal for the next. To solve this problem, our compiler generates different versions of optimized code based on different machine descriptions so that they can be deployed on different GPUs.

Table I lists the key parameters included in our proposed machine description files. Among them, the compiler applies vectorization based on the value of 'vectorization' parameter for the global memory version. If the vectorization is 2 or 4 , it means the preferred vector type is float 2 or float 4 , respectively. The compiler applies memory coalescing detection and code conversion based on CoalescedThread, which is the number of threads in a warp required for coalesced memory accesses. 'ThreadInBlock' defines the maximum thread number of one thread block. When the compiler applies the thread block merge step, the compiler uses ThreadInBlock/4 as an initial number of threads in a thread block. Another important parameter is RegisterInMP, which limits the aggressiveness of thread merge. The compiler chooses the register usage such that at least two thread blocks can be put in one MP. CacheLineSize is used to determine the block dimension for the texture memory version, as discussed in Section 3.5.1. Other parameters are used to check if current optimization step produces valid code. For example, when the compiler applies code conversion for memory coalescing and thread 


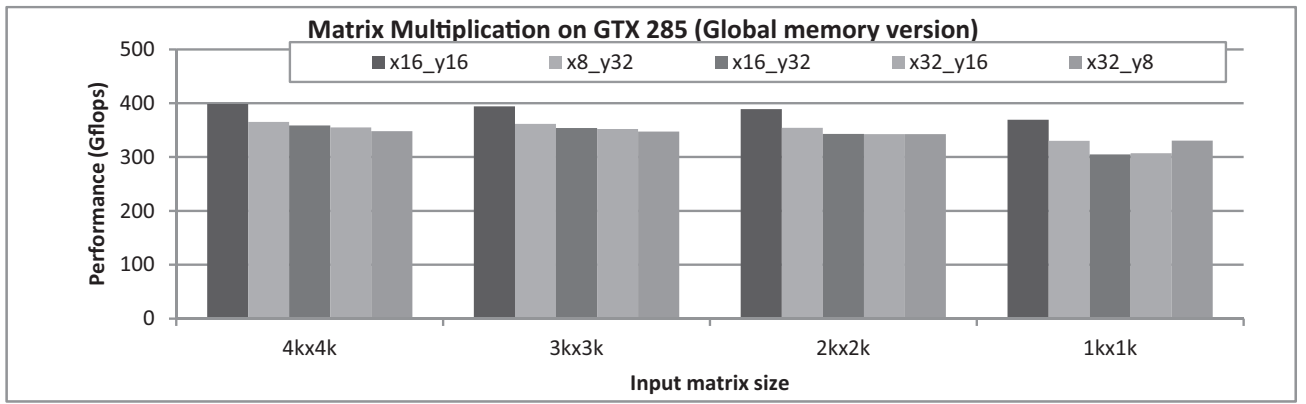

Fig. 13. The performance impact (GTX 285) of the number of merged threads/thread blocks. xN_yM means that $N$ thread blocks merged along the $X$ direction and $M$ threads merged along the $Y$ direction.

(block) merge, it always checks the shared memory usage with 'SharedMemoryInMP' to ensure that the shared memory usage does not exceed the hardware limitation.

\section{CASE STUDY}

\subsection{Case Study: Matrix Multiplication}

Matrix multiplication ( $\mathrm{mm}$ ) is a commonly used algorithm and there has been continuing effort to improve its performance [Nath et al. 2010]. The fine-tuned implementation in NVIDIA CUBLAS 2.2 has a throughput of 382 GFLOPS when computing the product of two $2 \mathrm{kx} 2 \mathrm{k}$ matrices on GTX 285 based on the work of [Volkov et al. 2008]. In the latest CUBLAS 3.2, the achieved performance is 713GFLOPS on GTX 480 [Nath et al. 2010]. In this section, we use matrix multiplication as an example to illustrate our compilation process for the global memory version.

The naïve kernel, that is, the input to our compiler, is shown in Figure 2(a). In the kernel, there are two input arrays, $a$ and $b$, from the global memory. The compiler converts the accesses to array $a$ into coalesced ones, as shown in Figure 6(a). Based on detected data sharing, the compiler determines that neighboring thread blocks along the $X$ direction can be merged to improve reuse of array $a$ and neighboring thread blocks along the $Y$ direction can be merged to improve memory reuse of array $b$. As the access to array $a$ is a R2S (read-to-shared memory), the compiler chooses to perform thread-block merge. As the access to array $b$ is R2R (read-to-register), the compiler chooses thread merge as discussed in Section 3.5. The next question is then how many thread blocks should be merged along either direction? As discussed in Section 4, the heuristic is to put at least 128 threads in each thread block and to generate different versions of kernel functions depending on the number of threads/thread blocks to be merged. Figure 13 shows the performance effect on GTX 285 of the number of merged threads/thread blocks in either direction. It can be seen that the optimal performance for different sizes of input matrices is achieved with merging 16 thread blocks along the $X$ direction and 16 threads along the $Y$ direction.

Since GTX 480 has larger shared memory and register file than GTX285, the compiler applies more aggressive thread merge as described in Section 3.5.3. For matrix multiplication, we show the performances of different number of merged threads along the $X$ and $Y$ directions in Figure 14. The number of merged thread blocks along the $X$ and $Y$ directions is set as 16. For comparison, we also include the version opt_285, which is optimized code for GTX 285. From the figure, we can see that opt_285 has the lowest performance and the version $\mathrm{x} 8 \mathrm{y} 4 \mathrm{4}$ achieves the highest performance for GTX 480 . 


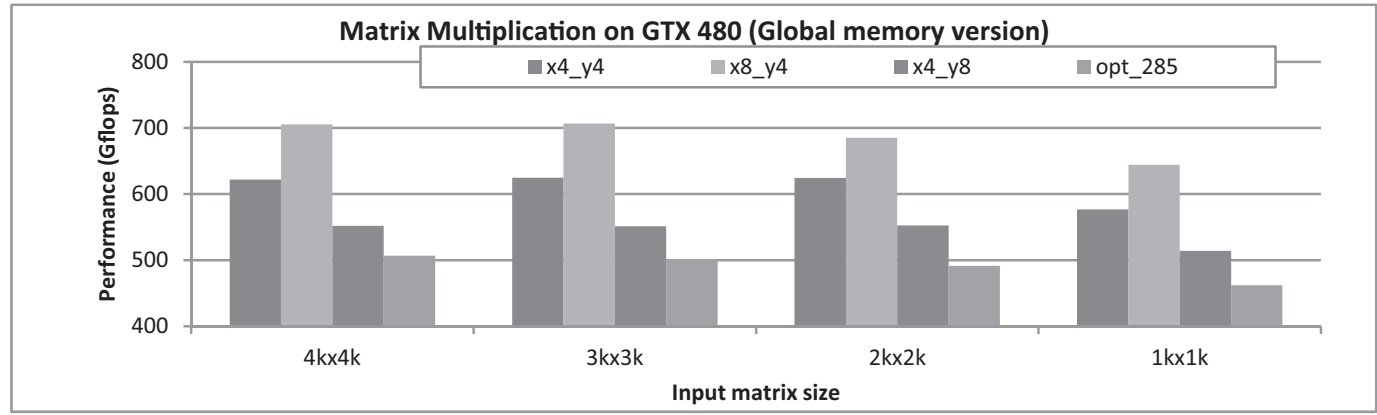

Fig. 14. The performance impact (GTX 480) of the number of merged threads. xN_yM means that $N$ threads merged along the $X$ direction and $M$ threads merged along the $Y$ direction. opt_285 is the best GTX 285 version running on GTX 480.

\subsection{Case Study: Matrix-Vector Multiplication}

Matrix-vector multiplication (mv) is one of BLAS2 applications. The algorithm has one input matrix, one input vector and one output vector. In this section, we use matrix vector multiplication as an example to illustrate our compilation process for the texture memory version.

The naïve implementation as the input of our compiler is shown in the Figure 2(b). For the texture memory version, the compiler applies vectorization first. In the kernel there are two texture memory inputs $a$ and $b$. The compiler applies loop-based vectorization to the kernel and the code after vectorization is shown in Figure 15(a). The compiler then performs thread merge along the $X$ and $Y$ directions to exploit data reuse. Because $\mathrm{mv}$ has only one dimension, the compiler tries to merge threads along the $X$ direction to reuse the vector $b$. Figure 15(b) shows the code after merging two threads along the $X$ direction. Since data reuse is very limited and thread merge may reduce thread-level parallelism, the compiler may also skip this thread step and then apply the partition camping elimination pass. The code after the transformation is shown in Figure 15(c). Figure 16 reports the performance on HD5870 for the different versions generated during the compilation process.

From Figure 16, it can be seen that vectorization has the highest performance impact. The thread merge step (either merging two or four threads along the $X$ direction) does not improve performance for $\mathrm{mv}$ due to the reduced number of threads. The impact of partition camping elimination depends on the data input sizes and improves the performance by up to $17 \%$ (on a $2 \mathrm{k} \times 2 \mathrm{k}$ matrix) on HD 5870 .

\section{EXPERIMENTS}

\subsection{Experimental Methodology}

We implemented the proposed compiler framework in Cetus, a source-to-source compiler infrastructure for C programs [Lee et al. 2003]. The CUDA language support in Cetus is ported from MCUDA [Stratton et al. 2008]. We also added the OpenCL language support so that our compiler can optimize naïve kernels written in either CUDA or OpenCL and generate the optimized kernels in either CUDA or OpenCL. The algorithms under study are listed in Table II. All the naïve kernels compute a single element at the position (idx, idy). The numbers of lines of code (LOC) of these naïve kernel functions are included in Table I to illustrate their programming complexity/ simplicity. Among the kernels, \#pragma is used in the reduction kernel to convey the information of input vector length and the actual output to the compiler. The purpose to know the actual output is to provide the flexibility to move the unnecessary global 
Table II. A List of the Algorithms Optimized with Our Compiler

\begin{tabular}{|l|l|c|}
\hline Algorithm & \multicolumn{1}{|c|}{$\begin{array}{c}\text { The size of input } \\
\text { matrices/vectors }\end{array}$} & $\begin{array}{c}\text { Num. of LOC } \\
\text { in the naïve kernel }\end{array}$ \\
\hline $\begin{array}{l}\text { transpose matrix vector } \\
\text { multiplication (tmv) }\end{array}$ & $1 \mathrm{kx} 1 \mathrm{k}$ to $4 \mathrm{kx} 4 \mathrm{k}$ (1k to 4k vec.) & 11 \\
\hline matrix mul. (mm) & $1 \mathrm{kx} 1 \mathrm{k}$ to $4 \mathrm{kx} 4 \mathrm{k}$ & 10 \\
\hline matrix-vector mul. (mv) & $1 \mathrm{kx} 1 \mathrm{k}$ to $4 \mathrm{kx} 4 \mathrm{k}$ & 11 \\
\hline vector-vector mul. (vv) & $1 \mathrm{k}$ to $4 \mathrm{k}$ & 3 \\
\hline reduction (rd) & $1-16$ million & 9 \\
\hline matrix equation solver (strsm) & $1 \mathrm{kx} 1 \mathrm{k}$ to $4 \mathrm{kx} 4 \mathrm{k}$ & 18 \\
\hline convolution (conv) & $4 \mathrm{kx} 4 \mathrm{k}$ image, $32 \times 32$ kernel & 12 \\
\hline matrix transpose (tp) & $1 \mathrm{kx} 1 \mathrm{k}$ to $8 \mathrm{kx} 8 \mathrm{k}$ & 11 \\
\hline Reconstruct image (demosaicing) & $1 \mathrm{kx} 1 \mathrm{k}$ to $4 \mathrm{kx} 4 \mathrm{k}$ & 27 \\
\hline $\begin{array}{l}\text { find the regional maxima } \\
\text { (imregionmax) }\end{array}$ & $1 \mathrm{kx} 1 \mathrm{k}$ to $4 \mathrm{kx} 4 \mathrm{k}$ & 26 \\
\hline
\end{tabular}

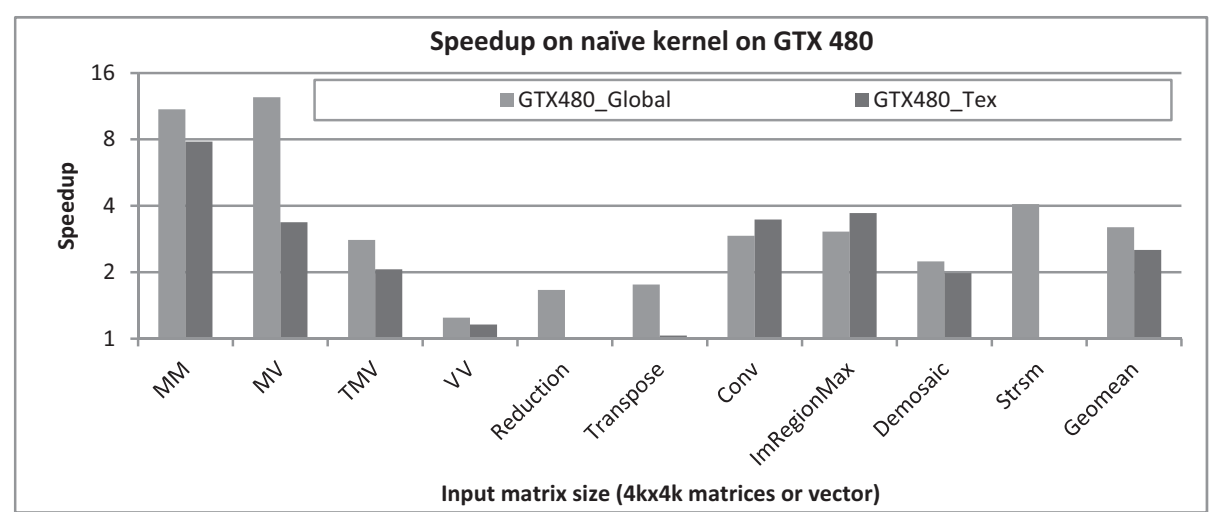

Fig. 17. The speedups of the optimized kernels over the naive ones. (the input to reduction is a vector of $16 \mathrm{M}$ floats) on GTX 480.

memory operations to shared memory operations. For example, if both A and B are defined as global memory arrays in a kernel and the compiler knows the $\mathrm{A}$ is the actual output, the compiler can replace memory accesses of B with shared memory accesses. The output of our compiler, that is, the optimized kernel, is compiled by the CUDA compiler, nvcc, or the OpenCL compiler to generate the GPU executable file. In our experiments, we used both NVIDIA GTX 285 and NVIDIA GTX 480 GPUs with CUDA SDK 3.2 and a 64-bit bit red hat enterprise Linux 5.4 operating system. For AMD/ATI HD 5870 GPUs, we used AMD/ATI Stream SDK 2.3 on a 32-bit Windows 7 operating system. Our compiler source code, the naïve kernels, and the optimized kernels are available at Yang and Zhou [2010]. Among the naïve kernels, reduction and strsm need to update the input array. Therefore we cannot take advantage of texture memories for these two benchmarks.

\subsection{Experimental Results}

In our first experiment, we examine the effectiveness of our compiler optimizations. Figure 17 and Figure 18 show the speedups of the optimized global and texture memory kernels over the naïve ones running on GTX 480 and AMD/ATI HD 5870, respectively. For GTX 285, we only show the performance of the optimized global memory kernels in Figure 19, as the texture support (i.e., texture cache) on GTX 285 is worse than GTX 480 as discussed in Section 2 . These figures show that the compiler significantly improves the performance using the proposed optimizations (7.6 times on GTX 285 


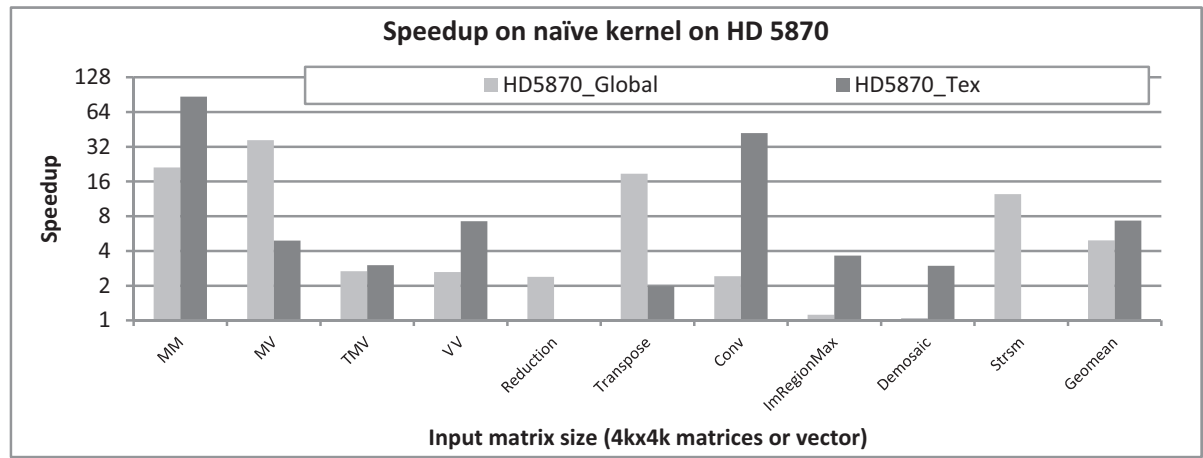

Fig. 18. The speedups of the optimized kernels over the naive ones. (the input to reduction is a vector of $16 \mathrm{M}$ floats) on HD 5870.

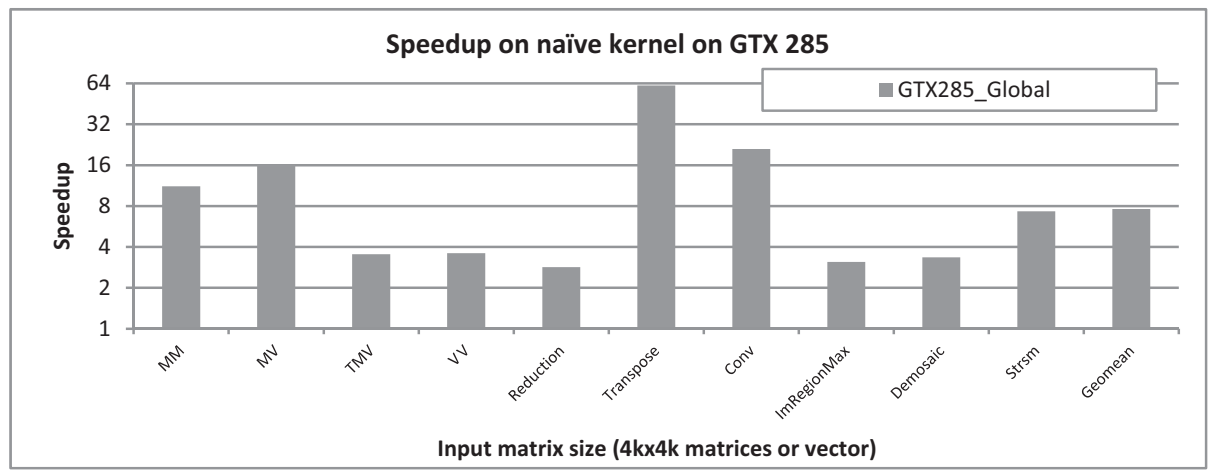

Fig. 19. The speedups of the optimized kernels over the naive ones. (the input to reduction is a vector of 16M floats) on GTX 285.

using global memories, 3.2 times on GTX 480 using global memories, 2.5 times on GTX 480 using texture memories, 4.9 times on HD 5870 using global memories, and 7.2 times on HD 5870 using texture memories on average with the geometric mean).

To better understand the achieved performance, we dissect the effect of each step of our compilation process and the results are shown in Figure 20 and Figure 21 for NVIDIA and AMD/ATI GPUs, respectively. The performance improvement achieved in each step is an average of all applications using the geometric mean. First, let us look at the texture memory version. On both HD 5870 and GTX 480, the vectorization has the highest impact on performance. Besides the benefit of memory bandwidth, the inter thread vectorization is essentially the same as the thread merge optimization by merging the neighboring threads, thereby reducing the significance of the thread merge step. Furthermore, for some benchmarks, the performance of the kernels after vectorization is very close to the theoretical peak performances as these algorithms are bounded by the bandwidth. The partition camping elimination deliver very little benefit. This is expected as the texture caches are well utilized and the impact of memory accesses is not critical. In fact, the step of partition camping elimination may actually incur performance degradation on GTX 480 due to the additional computation for mapping thread ids/thread block ids.

For the global memory version, since only intrathread vectorization is used for GTX 480 and GTX 285, this step has no effect on most of the kernels under study. In contrast, HD 5870 benefits significantly from vectorization, because HD 5870 has better support 


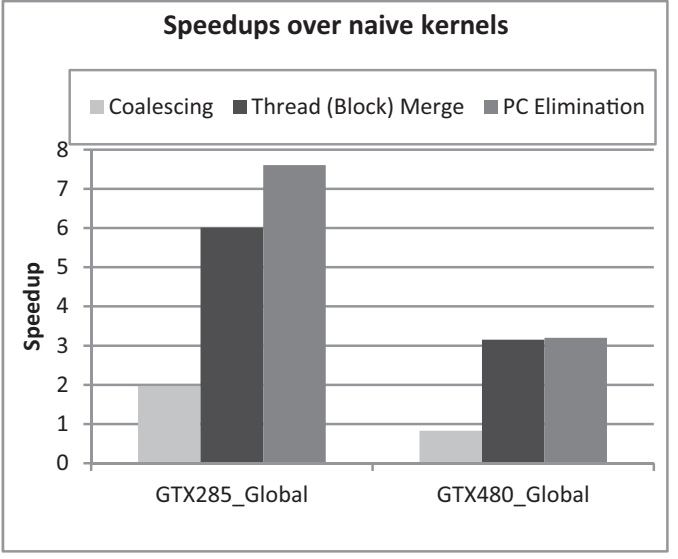

(a) global memory version

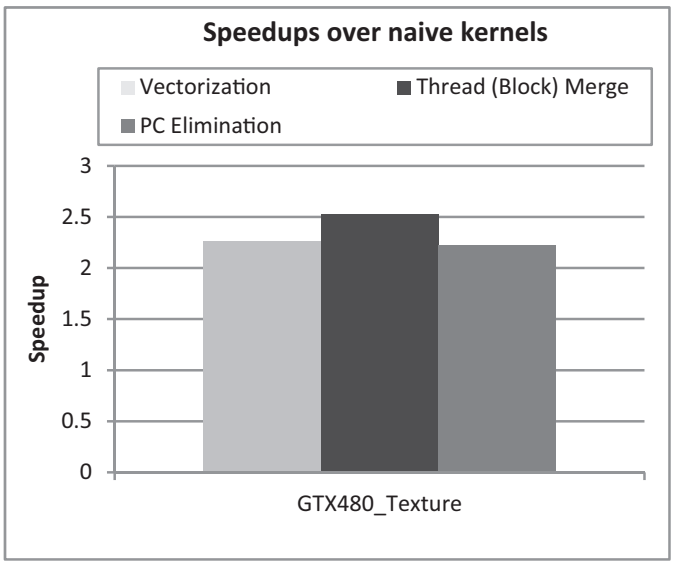

(b) texture memory version

Fig. 20. Performance improvement along the compilation process on GTX 480 and GTX 285 with $4 \mathrm{kx} 4 \mathrm{k}$ matrix/vector inputs.

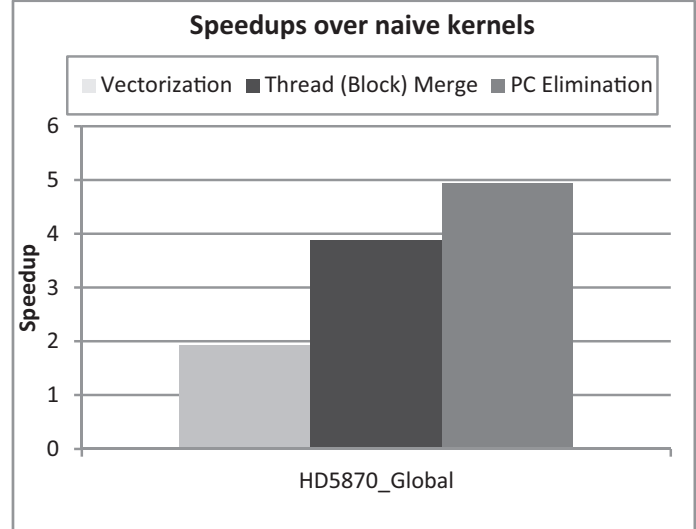

(a) global memory version

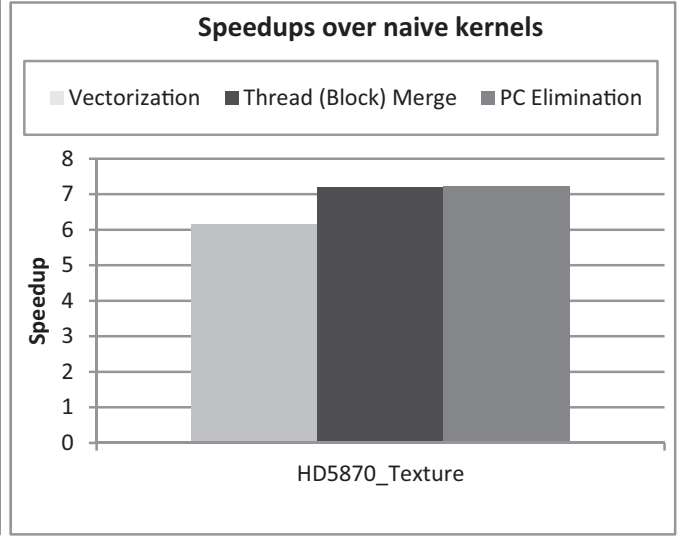

(b) texture memory version

Fig. 21. Performance improvement along the compilation process on HD 5870 with $4 \mathrm{kx} 4 \mathrm{k}$ matrix/vector inputs.

for float2 and float4 than float. We ignore the results of memory coalescing for HD 5870 . The reason is that after the memory coalescing step, a thread block has only 16 threads while one wavefront on HD 5870 has 64 threads. Such a small number of threads in a thread block results in severe resource underutilization and cannot show the actual impact of memory coalescing on HD5870. From the Figure 20(a), we can see that coalesced memory accesses are important for GTX 285, but not as much for GTX 480. The reason is due to the L1 data caches on GTX 480. The partition camping elimination has large impact on HD 5870 and GTX 285, but small impact on GTX 480. The reason is due to the input data sizes used in our experiments. For example, there is significant partition camping on HD 5870/GTX285 GPUs when transposing a $4 \mathrm{k} \times 4 \mathrm{k}$ matrix as they have 8 partitions. For the same input on GTX 480 which has 6 partitions, the accesses become more evenly distributed and eliminating partition camping has little effect. When transposing a $3 \mathrm{k} \times 3 \mathrm{k}$ matrix on GTX 480 , however, eliminating partition camping results in a $29 \%$ performance improvement. 


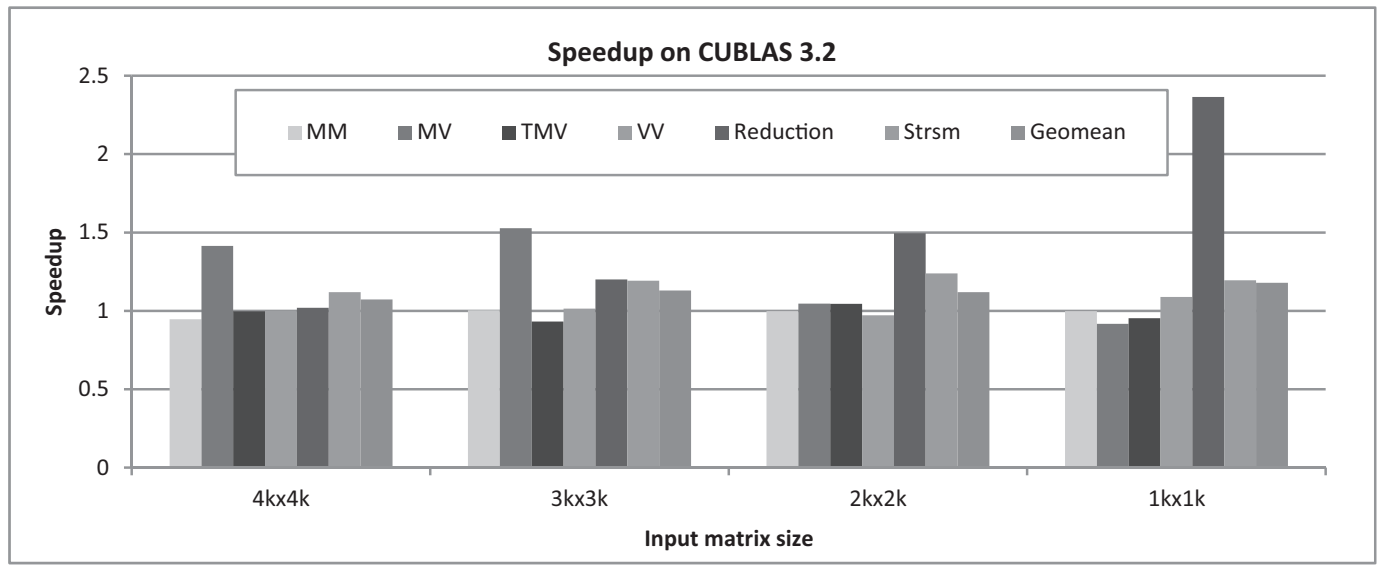

Fig. 22. Performance improvement of our optimized kernels over CUBLAS 3.2 implementations on GTX 285.

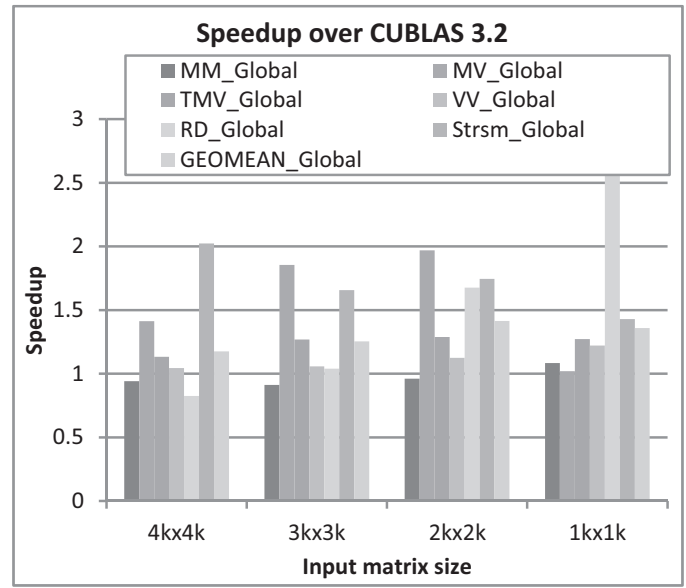

(a) global memory version

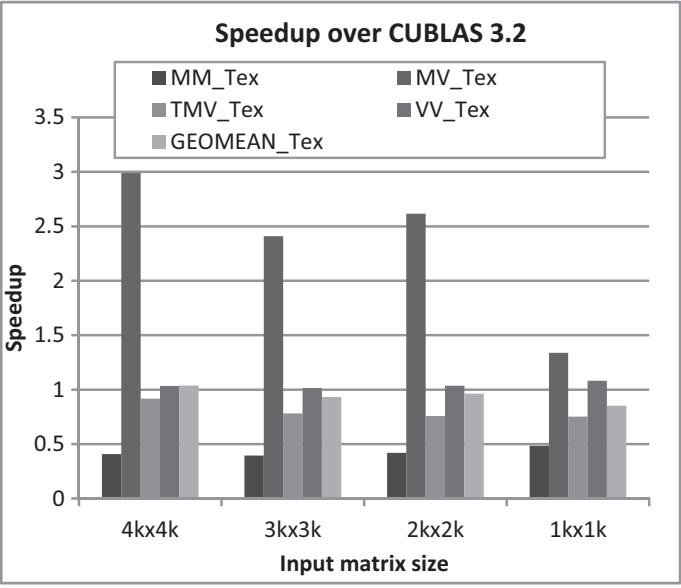

(b) texture memory version

Fig. 23. Performance improvement of our optimized kernels over CUBLAS 3.2 implementations on GTX 480 .

We did not include prefetching results, because prefetching shows little impacts[Yang et al. 2010]. The reason is that after thread/thread-block merge, the kernel consumes many registers. When allocating registers for prefetch, either the degree of thread merge must be reduced or the off-chip local memory may have to be used, resulting in degraded performance. Therefore, when registers are used up before prefetching, the prefetching step is skipped by our compiler.

Among the algorithms in Table II, six are implemented in the CUDA CUBLAS library. In the next experiment, we compare our optimized kernel with the highly tuned CUBLAS v3.2 on GTX 285 and GTX 480 . Figure 22 and Figure 23 show the performance comparison of the algorithms with different input sizes on GTX 285 and GTX 480, respectively. From Figure 23(a), we can see that the global memory version kernel optimized by our compiler achieves consistently better performance than CUBLAS 3.2 for transpose matrix vector multiplication (tmv), matrix vector multiplication $(\mathrm{mv})$, vector vector multiplication (vv), and matrix equation solver (strsm) for different input 


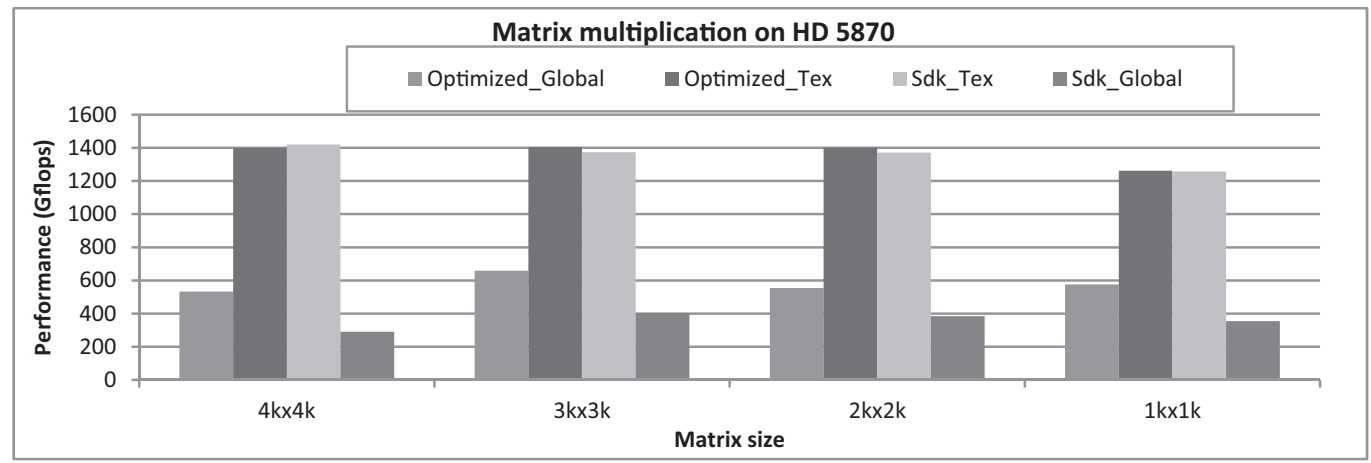

Fig. 24. Performance comparison between AMD/ATI Stream SDK and our optimized kernel on matrix multiplication.

sizes on GTX 480. For matrix multiplication $(\mathrm{mm})$ and reduction ( $\mathrm{rd}$ ), the performance of our optimized code is very close to CUBLAS 3.2 on GTX 480. On average (based on the geometric mean), our performance improvement of global memory version over CUBLAS varies from $17.5 \%$ to $41.4 \%$ for different input sizes on GTX 480 and varies from $7 \%$ to $17 \%$ on GTX 285 . Since the shared memory and L1 caches have better bandwidth than texture caches on GTX 480, the optimized texture memory version typically cannot compete with our optimized global memory version on GTX480. One exception is matrix vector multiplication, in which the shared memory is utilized to meet memory coalescing requirement in the global memory version. However, since there is no data reuse among different threads in this application, the texture cache turns out to be a more efficient way to achieve high bandwidth than coalesced memory accesses using the shared memory. In addition, the shared memory usage limits the number threads of each SM, which also limits the performance. Our texture memory version for vector-vector multiplication also outperforms CUBLAS v3.2 on GTX 480 but it is not as good as our optimized global memory version.

Customized matrix multiplication kernels for both the texture and global memory are provided in the AMD/ATI Stream SDK for AMD/ATI GPUs. We compare the performance of our optimized versions to the AMD/ATI Stream SDK on HD5870, as shown in Figure 24. From the figure, we can see our optimized texture memory version 'Optimized_tex' achieves very similar performance of the AMD/ATI Stream SDK texture memory version 'Sdk_Tex' and our optimized global memory version 'Optimized_global' outperforms the AMD/ATI Stream SDK global memory version 'Sdk_Global' for all the sizes. Between the texture and global memory versions, the texture memory versions achieve much higher performance than the global memory ones on HD5870 for matrix multiplication.

Figure 25 presents the performances of the BLAS2 benchmarks on HD 5870. Among the benchmarks, matrix-vector multiplication (mv) suits better with the texture cache, similar to our discussion of mv on GTX480. For transpose-matrix-vector multiplication, the texture memory version is better than the global memory version when the data size is large. On the other hand, vector-vector multiplication favors the global memory version when the data size is small. Overall, we can see that the texture memory/cache can be a very effective way to eliminate the memory coalescing issue and provide data reuse across threads/warps. It is application and device dependent to choose between the global memory and the texture memory for higher performance.

To study the effect of (intrathread) data vectorization for the global memory version on NVIDIA GPUs, we chose the reduction (rd) algorithm since rd is the only algorithm 


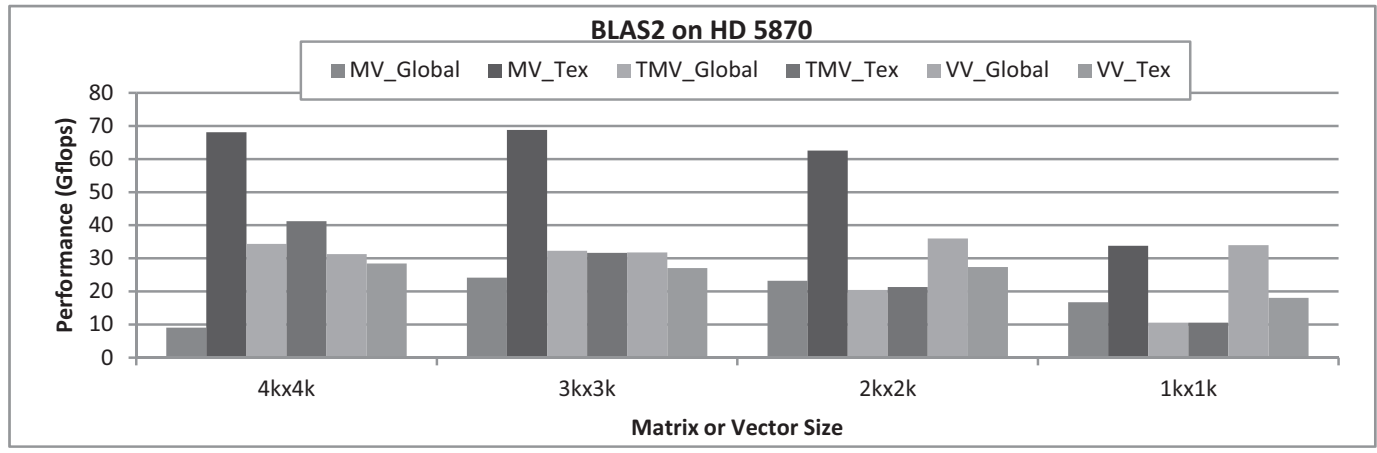

Fig. 25. Blas2 Performances on HD 5870.

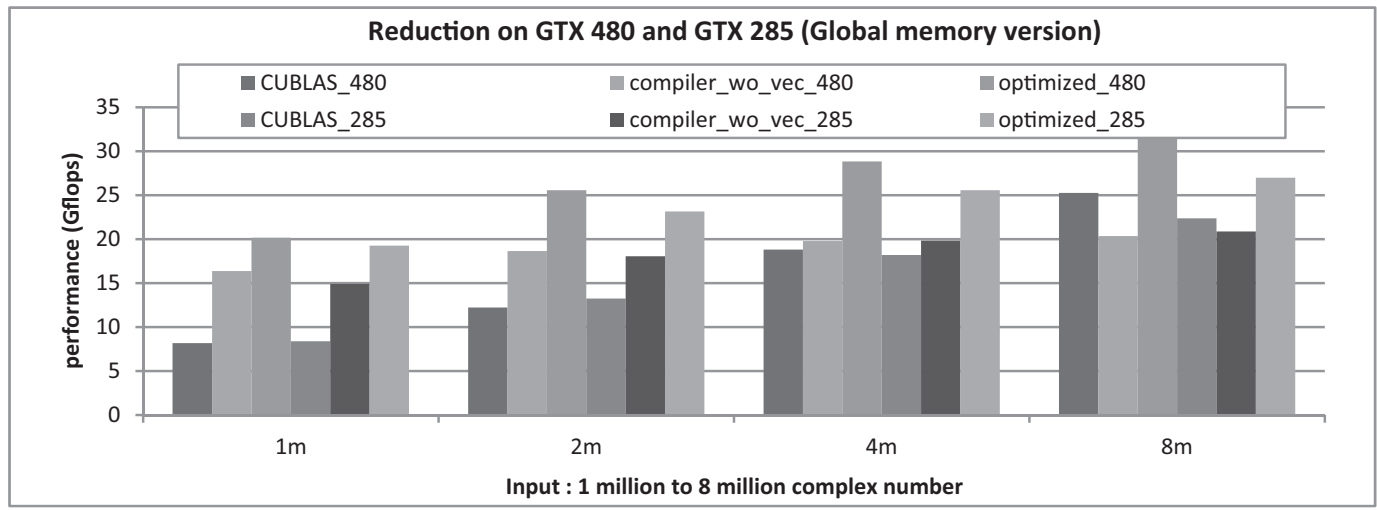

Fig. 26. The effect of data vectorization on reduction with complex number inputs.

in our study that has a corresponding version for complex numbers (CublasScasum) in CUBLAS. We changed the naïve kernel of rd to process complex numbers by using two float-type variables to read the real $(\mathrm{A}[2 * \mathrm{idx}])$ and imaginary $(\mathrm{A}[2 * \mathrm{idx}+1])$ parts of a complex number instead of a single float2 variable. Then, we optimized this naïve kernel with and without the data vectorization step. For different input sizes, we compared the performance of the two optimized kernels (labeled 'optimized_wo_vec' and 'optimized', respectively) and the results are show in Figure 26.

From Figure 26, we can see that data vectorization significantly improves the performance. One reason is the improved memory bandwidth due to the use of float2 data types as discussed in Section 2. Another reason is the side effect of memory coalescing. Without data vectorization, the compiler recognized that the array accesses to both real and imaginary parts $(\mathrm{A}[2 * \mathrm{idx}]$ and $\mathrm{A}[2 * \mathrm{idx}+1])$ are not coalesced. So, it uses shared memory as temporary storage to generate coalesced memory accesses as discussed in Section 3.3. In comparison, the accesses in the kernel after data vectorization, $\mathrm{A}[\mathrm{idx}]$, is coalesced. As a result, the data are directly loaded into registers for computation. Although the compiler uses the shared memory to improve memory reuse for both vectorized and un-vectorized versions, there are more shared memory accesses in the un-vectorized kernel optimized_wo_vec due to code transformation for coalescing. These extra shared memory accesses contribute to the performance differences between the optimized_wo_vec and optimized kernels.

Among all the kernels, transpose (tp) and matrix-vector multiplications (mv) exhibit the partition camping problem. Ruetsch and Micikevicius [2009] proposed diagonal 


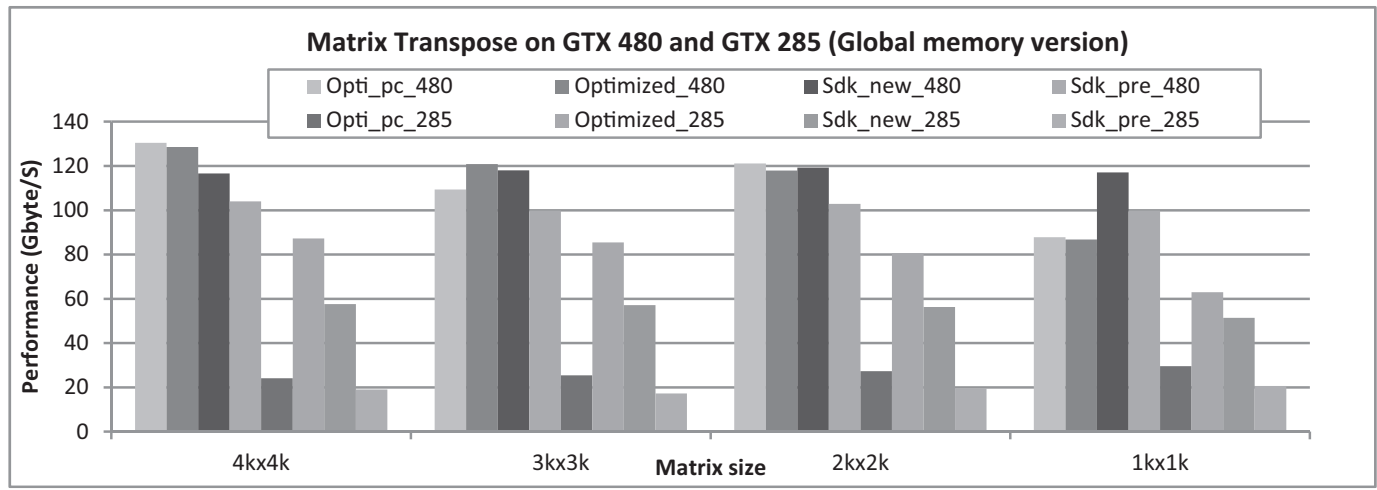

Fig. 27. Performance of matrix transpose using the optimized kernel for the global memory without partition camping elimination (labeled 'Opti_PC'), the optimized kernel for the global memory, and CUBLAS 3.2 on GTX 285 and GTX 480.

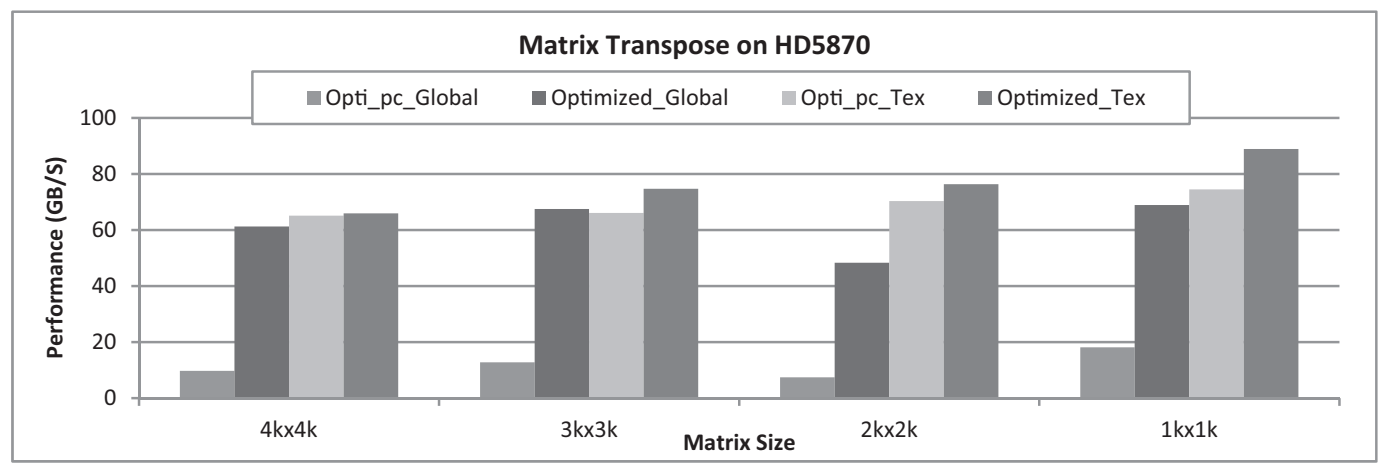

Fig. 28. Performance of matrix transpose using the optimized kernel for the global memory without partition camping elimination (labeled Opti_PC_global), the optimized kernel for the global memory (labeled optimized_global), the optimized kernel for the texture memory without partition camping elimination (labeled Opti_PC_tex), the optimized kernel for the texture memory (labeled 'optimized_tex') on HD 5870.

block reordering to address the issue with transpose and their implementation is included in the latest CUDA SDK. In Figure 27, we compare the performance of our optimized kernel (labeled 'optimized') with theirs (labeled 'SDK new') for tp on both GTX 285 and GTX 480 and we also include the previous CUDA SDK version for reference (labeled 'SDK pre'). Since tp does not have any floating point operations, the effective bandwidth is used. From Figure 27, it can be seen that although our compiler uses the same approach to eliminate partition camping, the remaining optimizations taken by our compiler result in better performance than the version in the latest SDK on both GTX 285 and GTX 480. Because GTX 480 has 6 partitions, the optimized version with partition camping elimination only outperforms the version without partition camping elimination when the input size is $3 \mathrm{k}$ by $3 \mathrm{k}$. We also observed similar performance impacts of partition camping on tp running on HD 5870, as shown in Figure 28, since HD5870 has 8 partitions. Note that, due to the texture cache, partition camping has much smaller impact than the global memory version.

In mv, the thread blocks are in one dimension. Therefore, diagonal block reordering cannot be applied. Our compiler uses the address offset approach described in Section 3.7 and the results are shown in Figure 29, Figure 30 and Figure 31 for GTX 285, GTX 480, and HD5870, respectively. It can be seen that for certain input sizes, 


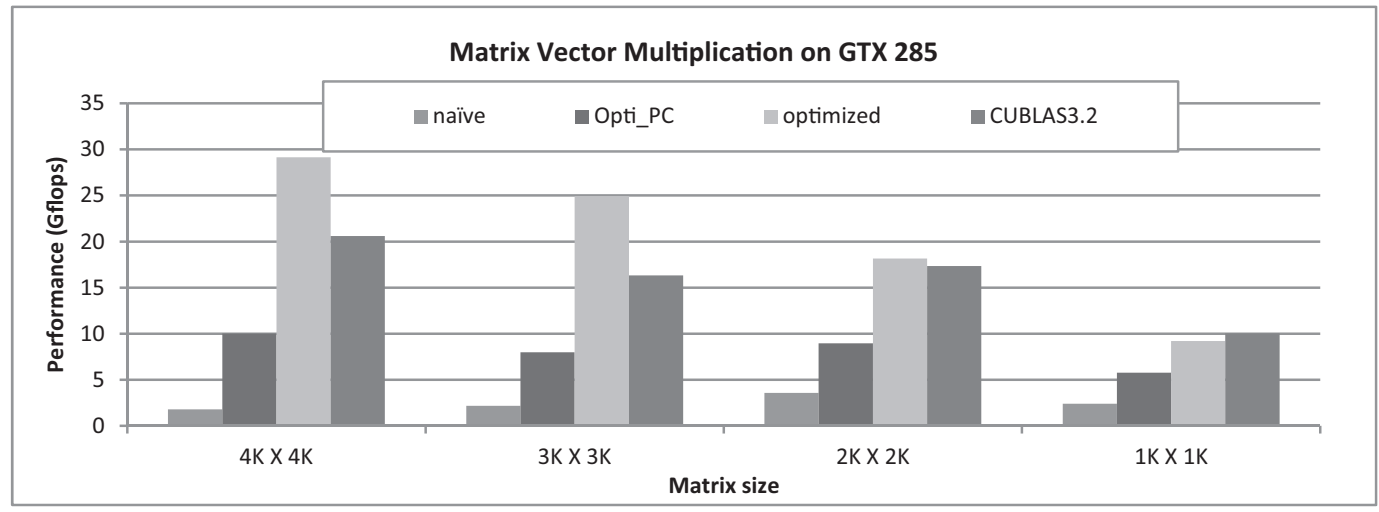

Fig. 29. Performance of mv using the naïve kernel, the optimized kernel without partition camping elimination (labeled Opti_PC), the optimized kernel, and CUBLAS 3.2 on GTX 285.

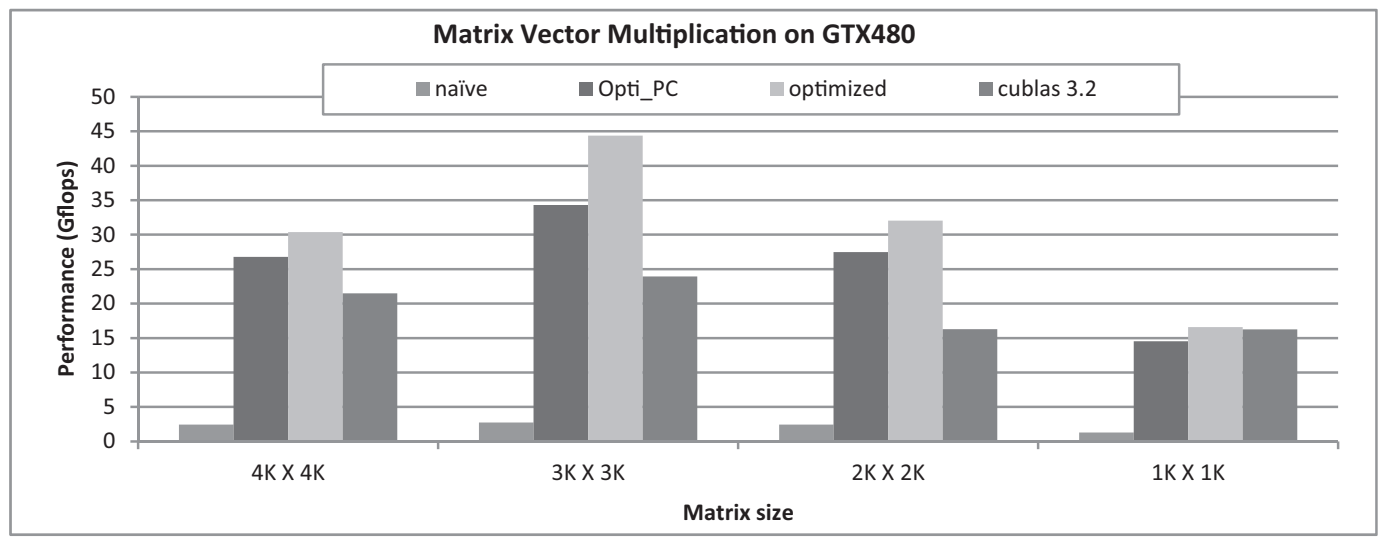

Fig. 30. Performance of mv using the naïve kernel, the optimized kernel without partition camping elimination (labeled Opti_PC), the optimized kernel, and CUBLAS 3.2 on GTX 480.

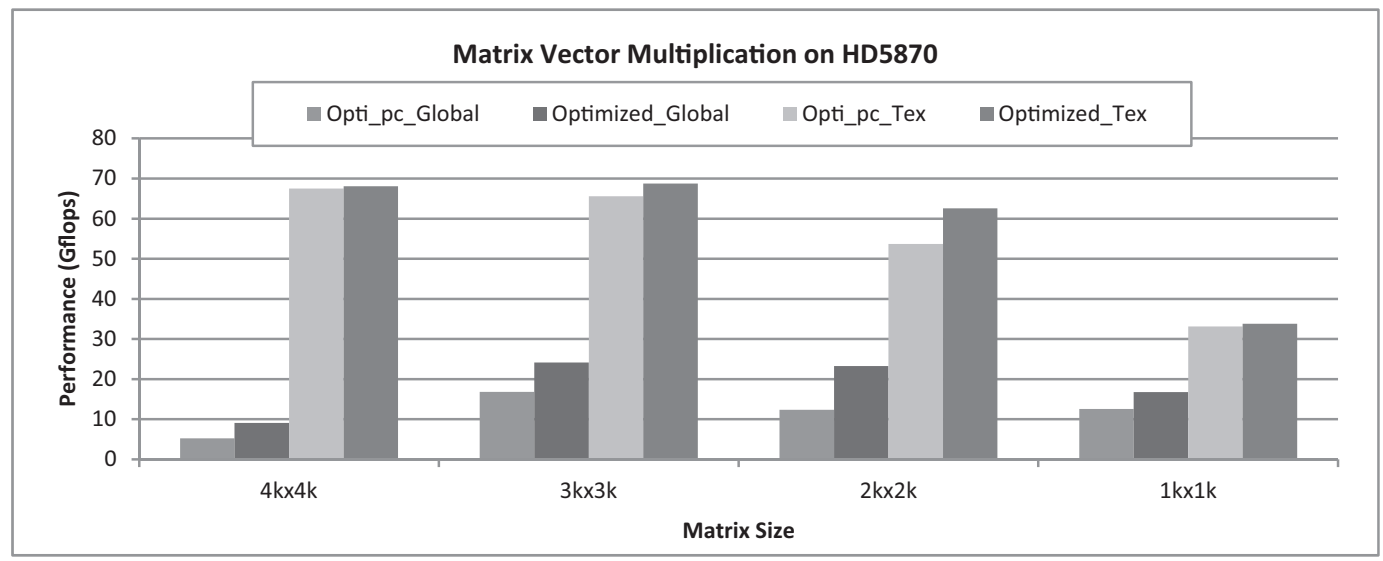

Fig. 31. Performance of mv using the optimized kernel without partition camping elimination (labeled Opti_PC), the optimized kernel on HD 5870. 
even without partition camping elimination, our optimized kernel (labeled Opti_PC) already achieves better performance than CUBLAS and eliminating partition camping (labeled optimized) further improves the performance. Similar to transpose, the eliminating partition camping has biggest impact on GTX 480 when the input size is $3 \mathrm{k}$ by 3k. On HD 5870 and GTX 285, as both GPUs have 8 partitions, eliminating partition camping has high performance impact due to the input data sizes being a multiple of $2 \mathrm{kB}$ (partition size of $256 \mathrm{~B} \times 8$ partitions).

In summary, our experimental results show that our optimizing compiler generates very high quality code for different GPGPU architectures and often achieves superior performance even compared to the manually optimized code in CUDA CUBLAS, CUDA SDK and AMD/ATI Stream SDK.

\section{LIMITATIONS}

Although the proposed compiler can dramatically improve the performance over naïve kernel functions, the fundamental limitation is that it cannot change the algorithm structure. Instead, our compiler can be used to facilitate algorithm-level exploration. The reasons are twofold. First, developers can leverage our aggressive compiler optimizations so that they do not need to optimize their implementations of each candidate algorithm. Second, the relatively good understandability of our optimized code may give a hint of what algorithms are better suited. Our compiler provides the generated code from each compilation step and it is relatively easy to understand what/how code transformations have been performed. Based on such understanding, a programmer may revise her naïve code for higher performance. Taking 1D fast Fourier transform (FFT) as an example, when the naïve kernel (50 lines of code) simply uses 2-point FFT in each step of the Cooley-Tukey algorithm [Cooley and Tukey 1965], the throughput is 24 GLOPS for computing the FFT of 220 complex numbers on GTX 285. Our compiler optimizes the naïve kernel by merging threads and the resulting kernel computes 8-point FFT in each step, which delivers a throughput of 41 GFLOPS, significantly better than CUFFT 2.2 (26GFLOPS). The compiler generated 8-point FFT version, however, is not as good as a naïve implementation of 8-point FFT (113 lines of code with a throughput of 44 GFLOPS). The reason is that the compiler generated version uses multiple 2-point FFT calculations for an 8-point FFT. On the other hand, as our compiler generated code is reasonably understandable, it serves as a good guideline for algorithm exploration: changing the naïve kernel from 2-point FFT to 8-point FFT, for which the compiler can further optimize the performance to achieve 59 GFLOPS. More elaborate algorithm-level development by Govindaraju et. al. [2008] as well as the one used in CUFFT2.3 achieves even higher performance (89 GFLOPS), indicating that our compiler facilitates but cannot replace intelligent algorithm-level exploration. Finally, in the present stage, our compiler assumes that the workload of each thread is similar and cannot handle the program very well if the workload can vary significantly due to control flow in different threads (i.e., index dependent workloads). One possible solution is a work item queue to balance the workload of each thread, which can be treated as another type of thread merge. We leave it as our future work.

\section{RELATED WORK}

CUDA [NVIDIA 2010] provides a relatively simple programming model to application developers. However, many hardware details are exposed since it is critical to utilize the hardware resources efficiently in order to achieve high performance. Given the nonlinear optimization space, optimizing GPGPU programs has been shown to be highly challenging [Ryoo et al. 2008a]. To relieve this task from developers, there has been some recent work on compiler support for GPGPU optimization. Ryoo et al. [2008b] defined performance metrics to prune the optimization spaces. G-ADAPT 
[Liu et al. 2009] is a compiler framework to search and predict the best configuration for different input sizes for GPGPU programs. Compared to our proposed approach, this compiler takes the optimized code and aims to adapt the code to different input sizes, while ours optimizes the naïve kernel functions.

One closely related work to ours is the optimizing compiler framework for affine loops by Baskaran et al. [2008]. Their compiler uses a polyhedral model to empirically search for best loop transformation parameters, including the loop tiling sizes and unrolling factors. It is reported that their compiler achieves similar performance to CUBLAS1.0 for matrix multiplication and better performance for other kernels. In comparison, our proposed compiler also uses empirical search to determine the best parameters to merge threads/thread blocks. The difference is that we propose a novel way to achieve the effect of loop tiling and loop unrolling. In our proposed approach, we start from the finest-grain work item and aggregate work items together to exploit data reuse through registers and share memory. This approach fits particularly well with GPGPU programming models where work items are typically defined in a 2D/3D grid and aggregating work items usually bears a clear physical meaning in terms of the workload of each thread and each thread block. In addition, we propose explicit rules to check memory coalescing and approaches to convert noncoalesced accesses into coalesced ones. For the applications that we studied, including matrix multiplication, our compiler achieves much better performance (superior or close to CUBLAS 3.2, which is significantly improved over CUBLAS 2.2 and CUBLAS 1.0). In addition, the loop transformed code generated based on polyhedral models is often quite complex [Pouchet et al. 2007] while our optimized code has relatively good understandability.

Our compiler shares a common goal with CUDA-lite [Ueng et al. 2008]: the user provides a kernel function which only uses the global memory and the compiler optimizes its memory usage. In CUDA lite, the compiler uses the programmer provided annotation to improve memory coalescing. It also performs loop tiling to utilize shared memory. In comparison, our compiler does not require user annotation. More importantly, not only does our compiler improve memory coalescing but also effectively achieves data sharing with the proposed thread/thread-block merge techniques. In addition, our compiler distinguishes memory reads based on their target, the register or the shared memory, to make best use of either type of resource for data reuse.

One interesting way to automatically generate GPGPU programs is to translate OpenMP programs to CUDA programs [Lee et al. 2009]. Our proposed compiler is complementary to this work as it can be used to further optimize the CUDA kernel functions generated from OpenMP programs.

\section{CONCLUSIONS}

In this article, we present a compiler framework to optimize GPGPU programs. The key idea is to present a simplified GPU hardware abstraction model to application developers so that they can focus on extracting fine-grain data-level parallelism from their applications rather than working on detailed performance optimizations. The extracted fine-grain data-level parallelism is captured as the naïve kernel input to our compiler. We propose a set of novel compiler techniques to improve GPU memory usage and distribute workload in threads and thread blocks. Our compiler generates two optimized kernels, one for global memories and the other for texture memories. Our experimental results show that the optimized code achieves very high performance, often superior to manually optimized programs.

\section{REFERENCES}

Aho, A. V., Sethi, R., and Ullman, J. D. 1986. Compilers: Principles, Techniques, and Tools. Addison-Wesley, Upper Saddle River, NJ. 
AMD, INC. 2011. AMD Accelerated Parallel Processing OpenCL Programming Guide 2.4.

Baghsorkhi, S. S., Delahaye, M., Patel, S. J., Gropp, W. D., and Hwu, W. W. 2010. An adaptive performance modeling tool for GPU architectures. In Proceedings of the 15th ACM SIGPLAN Symposium on Principles and Practice of Parallel Computing (PPOPP'10). ACM, 105-114.

Baskaran, M. M., Bondhugula, U., Krishnamoorthy, S., Ra-manujam, J., Rountev, A., and Sadayappan, P. 2008. A compiler framework for optimization of affine loop nests for GPGPUs. In Proceedings of the 22nd Annual International Conference on Supercomputing (ICS'08). ACM. 225-345.

Cooley, J. AND Tukey, J. W. 1965. An algorithm for the machine calculation of complex Fourier series. Math. Comput. 19, 297-301.

Fusimoto, N. Faster matrix-vector multiplication on GeForce 8800 GTX. 2008. In Proceedings of the IEEE International Parallel \& Distributed Processing Symposium (IPDPS'08). IEEE, 1-8.

Govindaraju, N., Lloyd, B., Dotsenko, Y., Smith, B., and Manferdelli, J. 2008. High performance discrete Fourier transforms on graphics processors. In Proceedings of the International Conference for High Performance Computing, Networking, Storage and Analysis (SC'08). IEEE. 1-12.

HonG, S. AND KIM, H. 2009. An analytical model for GPU architecture with memory-level and thread-level parallelism awareness. In Proceedings of the 36th International Symposium on Computer Architecture (ISCA'09). ACM.

LeE, S.-I., Johnson, T., AND Eigenmann, R. 2003. Cetus-An extensible compiler infrastructure for source-tosource transformation. In Proceedings of Workshops on Languages and Compilers for Parallel Computing (LCPC'03). 539-553.

Lee, S., Min, S.-J., And Eigenmann, R. 2009. OpenMP to GPGPU: A compiler framework for automatic translation and optimization. In Proceedings of the 14th ACM SIGPLAN Symposium on Principles and Practice of Parallel Computing (PPOPP'09). ACM, 101-110.

LiU, Y., ZHANG, E. Z., AND SHEN, X. 2009. A cross-input adaptive framework for GPU programs optimization. In Proceedings of IEEE International Parallel \& Distributed Processing Symposium (IPDPS'09). IEEE, $1-10$.

Nath, R., Tomov, S., and DongarRa, J. 2010. An improved MAGMA GEMM for Fermi GPUs. Tech. rep. UT-CS-10-655. University of Tennessee Computer Science.

NVIDIA, Inc. 2010. NVIDIA CUDA C Programming Guide 3.2.

OPENCL. http://www.khronos.org/opencl/.

Pouchet, L.-N., Bastoul, C., Cohen, A., And Vasilache, N. 2007. Iterative optimization in the polyhedral mode: Part I, On dimensional time. In Proceedings of International Symposium on Code Generation and Optimization (CGO’07). ACM, 144-156.

Ruetsch, G. AND Micikevicius, P. 2009. Optimize matrix transpose in CUDA. http://developer.download. nvidia.com/compute/cuda/sdk/website/C/src/transpose/doc/MatrixTranspose.pdf.

Ryoo, S., Rodrigues, C. I., Baghsorkhi, S. S., Stone, S. S., Kirk, D. B., and Hwu, W. W. 2008a. Optimization principles and application performance evaluation of a multithreaded GPU using CUDA.In Proceedings of the 13th ACM SIGPLAN Symposium on Principles and Practice of Parallel Programming (PPOPP'08). ACM, 73-82.

Ryoo, S., Rodrigues, C. I., Stone, S. S., Baghsorkhi, S. S., Ueng, S., Stratton, J. A., and Hwu, W. W. $2008 \mathrm{~b}$. Optimization space pruning for a multithreaded GPU. In Proceedings of the International Symposium on Code Generation and Optimization (CGO'08). ACM.

Stratton, J. A., Stone, S. S., AND Hwu, W. W. 2008. MCUDA: An efficient implementation of CUDA kernels for multi-core CPUs. In Proceedings of the 21st International Workshop on Languages and Compilers for Parallel Computing (LCPC'08). 16-30.

Ueng, S., Lathara, M., Baghsorkhi, S. S., and Hwu, W. W. 2008. CUDA-lite: Reducing GPU programming Complexity, in Proceedings of the 21st International Workshop on Languages and Compilers for Parallel Computing (LCPC'08). 1-15.

Volkov, V. ANd Demmel, J. W. Benchmarking GPUs to tune dense linear algebra. 2008. In Proceedings of the International Conference for High Performance Computing (SC'08), ACM. 1-11.

YANG, Y., XIANG, P., Kong, J., AND ZHou, H. 2010. A GPGPU Compiler for Memory Optimization and Parallelism Management. In Proceedings of the ACM SIGNPLAN 2010 Conference on Programming Language Design and Implementation (PLDI'10). ACM, 86-97.

YANG, Y. AND ZHOU, H. 2010. GPGPU compiler. http://code.google.com/p/gpgpucompiler/.

Received March 2011; revised July 2011; accepted September 2011 